\title{
USP16-mediated deubiquitination of calcineurin A controls peripheral T cell maintenance
}

\author{
Yu Zhang, ${ }^{1,2}$ Rong-bei Liu, ${ }^{2}$ Qian Cao, ${ }^{2}$ Ke-qi Fan, ${ }^{1}$ Ling-jie Huang, ${ }^{2}$ Jian-shuai Yu, ${ }^{1}$ Zheng-jun Gao, ${ }^{1}$ Tao Huang, ${ }^{1}$ Jiang-yan Zhong, ${ }^{1}$ \\ Xin-tao Mao, ${ }^{1}$ Fei Wang, ${ }^{1}$ Peng Xiao, ${ }^{2}$ Yuan Zhao, ${ }^{2}$ Xin-hua Feng, ${ }^{1}$ Yi-yuan Li, ${ }^{1}$ and Jin Jin ${ }^{1,2,3}$ \\ 'MOE Key Laboratory of Biosystems Homeostasis and Protection, Life Sciences Institute, Zhejiang University, Hangzhou, China. ${ }^{2}$ Sir Run Run Shaw Hospital, College of Medicine Zhejiang University,

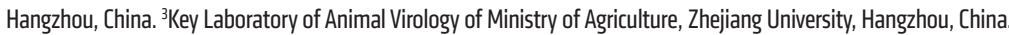

\begin{abstract}
Calcineurin acts as a calcium-activated phosphatase that dephosphorylates various substrates, including members of the nuclear factor of activated T cells (NFAT) family, to trigger their nuclear translocation and transcriptional activity. However, the detailed mechanism regulating the recruitment of NFATs to calcineurin remains poorly understood. Here, we report that calcineurin A (CNA), encoded by PPP3СB or PPP3CC, is constitutively ubiquitinated on lysine 327, and this polyubiquitin chain is rapidly removed by ubiquitin carboxyl-terminal hydrolase 16 (USP16) in response to intracellular calcium stimulation. The K29-linked ubiquitination of CNA impairs NFAT recruitment and transcription of NFAT-targeted genes. USP16 deficiency prevents calcium-triggered deubiquitination of CNA in a manner consistent with defective maintenance and proliferation of peripheral T cells. T cell-specific USP16 knockout mice exhibit reduced severity of experimental autoimmune encephalitis and inflammatory bowel disease. Our data reveal the physiological function of CNA ubiquitination and its deubiquitinase USP16 in peripheral T cells. Notably, our results highlight a critical mechanism for the regulation of calcineurin activity and a novel immunosuppressive drug target for the treatment of autoimmune diseases.
\end{abstract}

\section{Introduction}

Calcineurin (protein phosphatase 2B) is the only serine/threonine protein phosphatase regulated by intracellular calcium (1). Calcineurin participates in a variety of signal transduction pathways regulated by cytoplasmic $\mathrm{Ca}^{2+}$, and performs various physiological functions by interacting with distinct substrates (2). As a major substrate of calcineurin, nuclear factor of activated T cells (NFAT) is widely distributed in various tissues and cell types (3). Activated calcineurin directly dephosphorylates NFATs in the cytoplasm and induces their translocation to the nucleus (4-6). In addition to dephosphorylating NFATs, calcineurin is recruited to the T cell receptor (TCR) signaling complex, where it reverses the inhibitory phosphorylation of lymphocyte-specific protein tyrosine kinase (LCK) on serine $59(7,8)$. Calcineurin-mediated signaling is involved in a diverse range of biological processes, including $\mathrm{T}$ cell maturation and reactivity (9). Calcineurin is also required for development of the nervous (10), skeletal muscle, and cardiovascular systems (11-13). Calcineurin is composed of a catalytic calcineurin A (CNA) subunit and a $\mathrm{Ca}^{2+}$-binding regulatory calcineurin $\mathrm{B}$ (CNB) subunit. CNA includes a calmodulin-binding (CaM-binding) domain and an autoinhibitory domain (AID). Once bound to $\mathrm{Ca}^{2+}, \mathrm{CNB}$ and $\mathrm{CaM}$ are thought to induce a structural change in the AID, thereby releasing its inhibitory effect on the catalytic activity of CNA (14). However, whether the release

Authorship note: Y. Zhang, RL, QC, and KF contributed equally to this work. Conflict of interest: The authors have declared that no conflict of interest exists. Copyright: (c) 2019, American Society for Clinical Investigation.

Submitted: July 25, 2018; Accepted: April 9, 2019; Published: May 28, 2019

Reference information: J Clin Invest. 2019;129(7):2856-2871.

https://doi.org/10.1172/JCl123801. of AID from the catalytic domain is sufficient for calcineurin activation remains poorly understood. Additionally, the lack of activation markers has hindered the discovery of more selective inhibitors of calcineurin.

A wide range of regulatory proteins has been reported to regulate calcineurin activity (15). A well-known family is the regulators of calcineurin (RCAN), which interacts with CNA via the hydrophobic pocket $(16,17)$. A-kinase anchor proteins (AKAPs) and Cabin 1 also exert inhibitory effects on activated calcineurin (18-20). Previous evidence has revealed that calcineurin can be phosphorylated by casein kinase I (CKI) and protein kinase C (PKC) $(21,22)$, but the physiological function of this phosphorylation remains unclear. In this study, we found that CNA is highly modified by nonproteolytic ubiquitination in resting $\mathrm{T}$ cells. The resulting polyubiquitin chain is rapidly removed in response to elevated calcium concentrations. The ubiquitination of CNA disrupts its interaction with NFATs. Furthermore, the deubiquitination of CNA is mediated by ubiquitin carboxyl-terminal hydrolase 16 (USP16), which selectively binds 2 isozymes of CNA encoded by PPP $3 C B(3 \mathrm{CB})$ and $P P P 3 C C ~(3 \mathrm{CC})$.

USP16 is a deubiquitinase (DUB) that has been found to be required for chromosomal segregation in mitosis (23). USP16 promotes the localization and maintenance of polo-like kinase 1 (PLK1) on kinetochores, which are important for proper chromosome alignment. USP16 can also deubiquitinate histone H2A and regulate gene expression when coupled with protein regulator of cytokinesis 1 (PRC1) $(24,25)$. Dysregulated expression of USP16 is associated with Down Syndrome (DS), a congenital disease characterized by the triplication of chromosome 21 (HSA21) (26). However, no evidence has clarified why children with DS have an increased risk of developing acute lymphoblastic leu- 
kemia (ALL) (27). Data obtained in this study demonstrate that peripheral $\mathrm{CD} 4^{+} \mathrm{T}$ cells from patients with distinct autoimmune diseases display elevated levels of USP16. We further present biochemical evidence showing that USP16 functions as a DUB of CNA in activated T cells and that USP16 deficiency results in impaired calcineurin activity. USP16 is recruited to CNA upon TCR stimulation and selectively removes K29-linked polyubiquitin chains from $3 \mathrm{CB}$ and $3 \mathrm{CC}$. USP16 acts as a critical regulator of $\mathrm{T}$ cell activation and $\mathrm{T}$ cell-mediated autoimmune diseases. $\mathrm{T}$ cell-specific USP16 knockout (USP16-KO) mice exhibit a severely reduced number of peripheral $\mathrm{T}$ cells coupled with diminished autoimmune symptoms. In contrast to previous findings (28), USP16 deficiency did not result in suppression at metaphase, but rather directly attenuated TCR-induced calcium signaling and NFAT activation in our study. Therefore, our findings demonstrate what we believe is a novel function and mechanism for USP16 in regulating mature $\mathrm{T}$ cell activation, and suggest that USP16 might serve as a novel therapeutic target in the treatment of $\mathrm{T}$ cell-mediated autoimmune diseases.

\section{Results}

Nonproteolytic ubiquitination represses calcineurin activity and NFAT recruitment. Calcineurin is known to be involved in $\mathrm{T}$ cell activation and calcium-dependent signal transduction (5). However, the detailed mechanism and modification indicating calcineurin activation remain poorly understood. Interestingly, our findings demonstrated that in murine primary $\mathrm{CD} 4^{+} \mathrm{T}$ cells, the catalytic subunit of CNA was rapidly deubiquitinated upon TCR or PMA/ionomycin $(\mathrm{P} / \mathrm{I})$ stimulation without any change in its protein level (Figure 1A and Supplemental Figure 1A; supplemental material available online with this article; https://doi. org/10.1172/JCI123801DS1). Consistently, human $\mathrm{CD}^{+}{ }^{+} \mathrm{T}$ cells obtained from the peripheral blood mononuclear cells (PBMCs) of healthy donors exhibited similar CNA deubiquitination after P/I stimulation for 5 minutes (Figure 1B and Supplemental Figure 1B). As shown in Figure 1C, calcineurin is a heterodimer of regulatory subunit $\mathrm{CNB}$ and catalytic subunit CNA, which can be encoded by any of 3 genes (PPP3CA, PPP3CB, and PPP3CC). To identify the potential modification sites on $C N A$, we evaluated ubiquitination levels obtained with distinct truncations of CNA. Taking $3 \mathrm{CB}$ as an example, we found that the polyubiquitination level was markedly reduced when the amino acids at positions 326-351 were deleted (Figure 1D and Supplemental Figure 1C). These results suggest that potential ubiquitination sites are located at residues 326-351. Ubiquitination usually results in the attachment of ubiquitin to lysine residues $(\mathrm{K})$ on substrate proteins or itself. Therefore, we analyzed the lysine residues in the sequence NNKAAVLKYENNVMNIRQFNCSPHPYW. As indicated in Figure 1E, we identified only 2 lysine residues K327 and K332 in this fragment. To confirm the ubiquitination sites of CNA, we isolated CNA from naive $\mathrm{CD} 4^{+}$ $\mathrm{T}$ cells and identified ubiquitination sites using mass spectrometry. As shown in Supplemental Figure 1E and Supplemental Table 3, mass spectrometry analysis revealed $\mathrm{K} 61$ and K327 was ubiquitinated. Additionally, $70 \%$ of the fragment NNKAAVLK was ubiquitinated. These data supported our conclusion that ubiquitination is a critical process for CNA to avoid its autoactivation. To further study the functional significance of CNA at these specific lysine residues, we substituted the lysine residues with arginine residues $(\mathrm{R})$ to generate point mutants of CNA. As shown in Figure $1 \mathrm{~F}$ and Supplemental Figure 1D, the K327R mutation resulted in the loss of ability of the fragment to be polyubiquitinated. Interestingly, K327 is conserved among all 3 CNA isozymes (Figure 1G) and among different species (Figure $1 \mathrm{H}$ ). Consistent with the findings obtained from $3 \mathrm{CB}$, mutation of $3 \mathrm{CA}$ or $3 \mathrm{CC}$ at this conserved lysine residue also resulted in a reduced level of ubiquitination (Supplemental Figure 1, F and G).

The NMR structure of $3 \mathrm{CB}$ revealed that $\mathrm{K} 327$ is located within a hydrophobic pocket that binds to the short linear motif (PxIxIT) of NFATs (Figure 1I). Thus, we hypothesized that CNA ubiquitination is potentially involved in its interaction with substrates. Coimmunoprecipitation (coIP) assays demonstrated that defective ubiquitination of CNA led to an elevated recruitment of NFAT2 (Figure 1J). The AID of CNA inhibits NFAT recruitment to the active site of CNA. However, K327 mutant of CNA did not affect the binding of AID with the catalytic domain (Supplemental Figure 1H). Collectively, these data indicate that deubiquitination of CNA at K327 is critical for NFAT recruitment in activated T cells.

USP16 is selectively associated with CNA encoded by $P P P 3 C B$ and PPP3CC. To identify the potential DUBs that regulate CNA deubiquitination, we screened for interactions between CNA and 46 individual DUBs in HEK293T cells. The coIP results indicated that only ectopic USP16 was associated with endogenous CNA (Figure 2A and Supplemental Figure 2A). As shown in previous studies, cysteine 205 (C205) in human USP16 is required for its catalytic activity (29-31). Transfection of wild-type USP16 (USP16-WT), but not the catalytically inactive USP16 ${ }^{\mathrm{C} 205 \mathrm{~s}}$ mutant (USP16-CI) resulted in higher P/I-induced NFAT activity in $\mathrm{CD}^{+} \mathrm{T}$ cells, as demonstrated by NFAT luciferase reporter assays (Figure 2B and Supplemental Figure 2B). In the absence of a stimulus, the coIP results showed no interaction between endogenous USP16 and CNA, whereas TCR ligation rapidly (within 2 minutes) induced USP16 binding to CNA (Figure 2C). We further determined the subcellular distribution of USP16 and its colocalization with CNA by confocal microscopy. In resting $\mathrm{CD}^{+}{ }^{+} \mathrm{T}$ cells, USP16 was mainly localized in the nucleus and separated from cytosolic CNA (Figure 2D). After TCR stimulation for 3 hours, significant colocalization of USP16 with CNA was observed in the cytoplasm, which was demonstrated by a markedly increased Pearson's correlation coefficient (Figure 2D and Supplemental Figure 2C). In cotransfected HEK293T cells, coIP assay results revealed that USP16 physically associated only with $3 \mathrm{CB}$ and $3 \mathrm{CC}$, but not 3CA (Figure 2E). To confirm the lack of association between USP16 and 3CA in T cells, we used a specific antibody against 3CA for coIP assay. We combined the lysates of peripheral $\mathrm{CD}^{+} \mathrm{T}$ cells and subjected them to IP using anti-CNA or anti-3CA antibody. In contrast to anti-CNA antibody, anti3CA antibody indicated no interaction between USP16 and 3CA in peripheral $\mathrm{CD}^{+} \mathrm{T}$ cells or thymocytes (Supplemental Figure 2D). The association of overexpressed USP16 with 3CB or 3CC was constitutive in HEK293T cells, which was different from that in mature T cells. Confocal images revealed that transfected USP16 was mainly distributed in the cytoplasm in the absence of stimulation, similar to what was observed in activated $\mathrm{T}$ cells (Supplemental Figure 2E). Additional ionomycin stimulation 
A

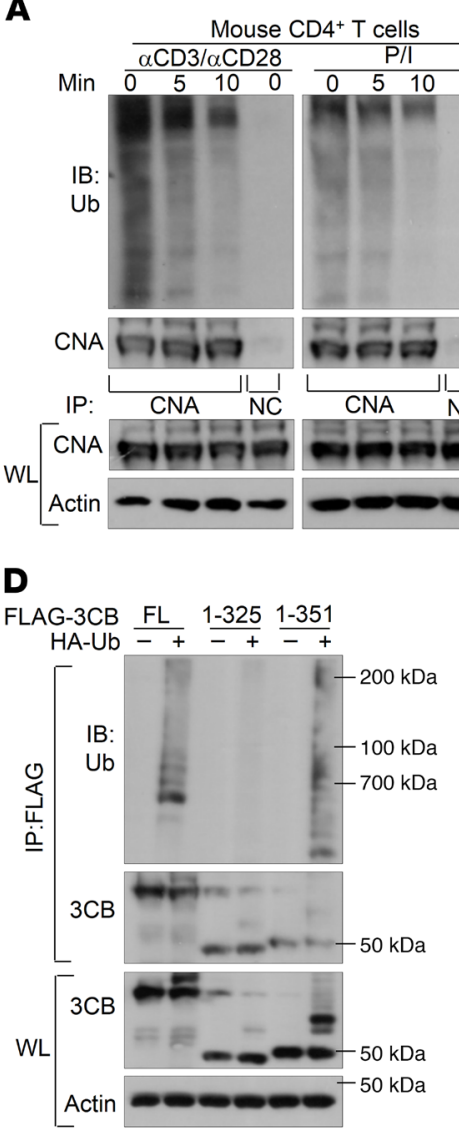

G

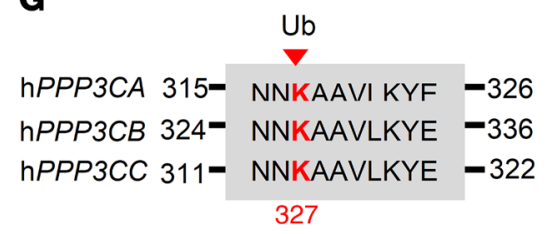

B
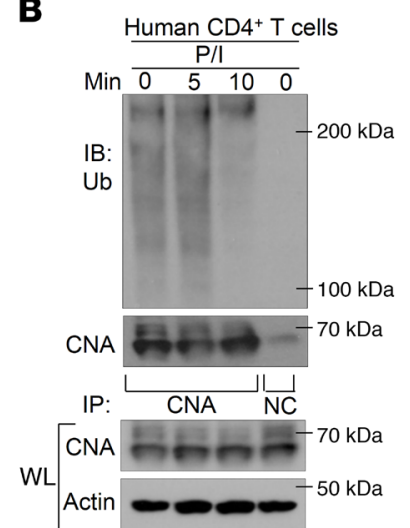

$\mathbf{F}$

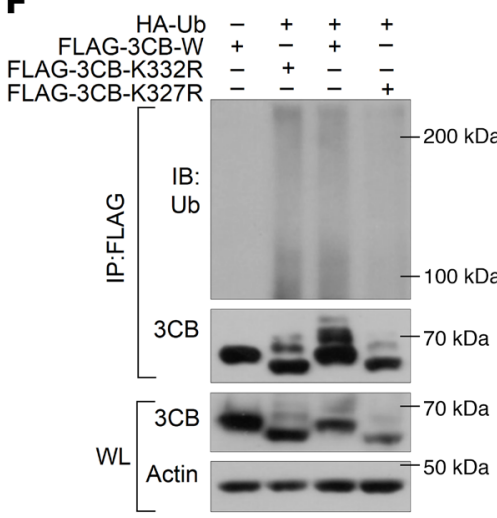

J

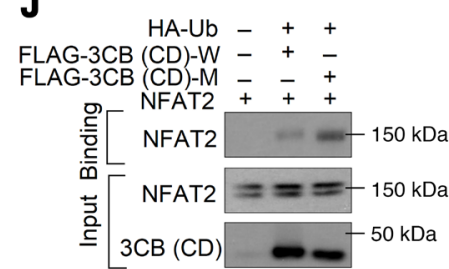

C
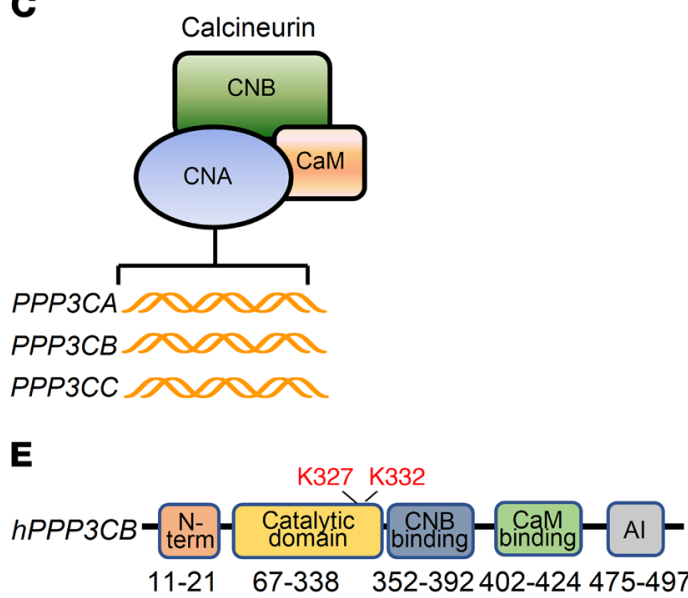

H

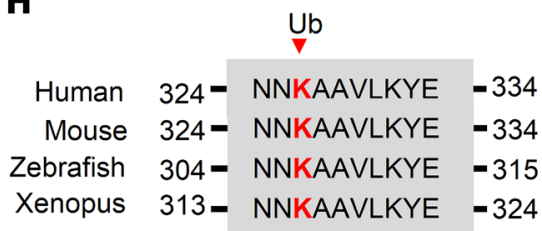

I

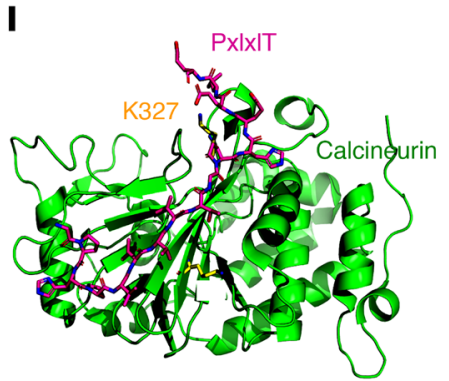

Figure 1. T cell activation is associated with the deubiquitination of calcineurin $\mathbf{A}$. (A) IB analysis of the deubiquitination of calcineurin $A$ (CNA) in mouse splenic CD4 ${ }^{+} \mathrm{T}$ cells upon $\alpha \mathrm{CD} 3 / \alpha \mathrm{CD} 28$ or PMA/ionomycin (P/I) stimulation. Whole-cell lysate (WL) was subjected to IP using anti-CNA or rabbit IgC control as nonspecific control (NC), followed by IB analysis of the ubiquitination (Ub) level. (B) Human CD4+ $T$ cells isolated from the peripheral blood of healthy donors were activated with P/I. The same Ub assay was performed as described in A. Immunoprecipitated CNA was detected by Trueblot. (C) Calcineurin is composed of calcineurin B (CNB) and CNA, and the latter is encoded by PPP3CA, PPP3CB, or PPP3CC. (D) HEK293T cells were transfected with a distinct truncation mutant of CNA encoded by PPPЗСB (ЗСB) along with HA-tagged Ub. WLs were subjected to IP using anti-FLAC antibody followed by Ub analysis. The WLs were also subjected to direct IBs (bottom 2 panels). (E) Schematic representation of the different domains of human 3CB. (F) Wild-type 3CB (3CB-W) and Ub site mutants were transfected into HEK293T cells. Whole-cell lysates were subjected to IP using anti-FLAG followed by Ub analysis. (C) Comparison of the amino acid sequences around potential Ub sites on human CNA catalytic subunit. (H) Sequence alignment of Ub sites on CNA orthologs of different species. (I) Crystal structure of the CNA: NFAT2 PxIxIT complex. The potential Ub sites are shown in yellow. (J) Interaction assay of in vitro translational NFAT2 with the ubiquitinated catalytic domain (CD) of WT or mutant 3CB, which was isolated from transfected HEK293T cells. Data are representative of 4 independent experiments with 4 mice in each group (A, B), 4 experiments (D, F), and 3 experiments (J).

only induced a weak enhancement of the interaction between USP16 and 3CB in these HEK293T cells (Supplemental Figure $2 \mathrm{~F}$ ). All of these data suggested that $\mathrm{P} / \mathrm{I}$ - or TCR-triggered translocation of USP16 from the nucleus to the cytoplasm is essential for its binding with CNA during $\mathrm{T}$ cell activation.

USP16 contains a peptidase domain and a zinc finger ubiquitin-binding domain (Znf-Ubp). To map the crucial domains of CNA and USP16 that are responsible for their interaction, we generated different truncated forms of USP16 (1-142 and 184-C) and 3CB (1-351, 1-401, 1-474, and 22-C). Results of co-IP using HEK293T cells revealed that the association between CNA and USP16 was dependent on the catalytic domain of CNA and the peptidase domain of USP16 (Figure 2, F and G). We also identified 5 consecutive amino acids, TMIEV, which are conserved between $3 \mathrm{CB}$ and $3 \mathrm{CC}$, but not in 3CA (Figure 2, $\mathrm{H}$ and I). If these 5 amino acids were deleted, no association was detected between USP16 and 3CB $\triangle 86-91$ (Figure 2J). Thus, TCR engagement triggers the selective binding of USP16 to $3 \mathrm{CB}$ or $3 \mathrm{CC}$ in activated T cells. 
A
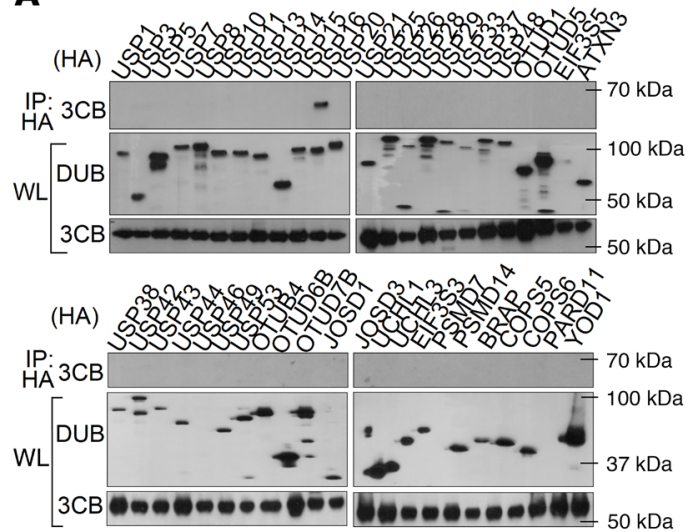

E

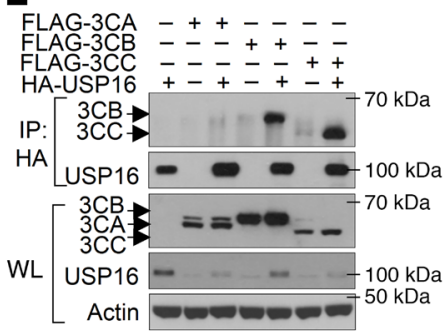

$\mathbf{F}$

\begin{tabular}{|c|c|c|}
\hline $\mathrm{FL}$ & UBP & Peptidase domain \\
\hline-142 & U४िP & \\
\hline $34-C$ & & Peptidase domain \\
\hline
\end{tabular}

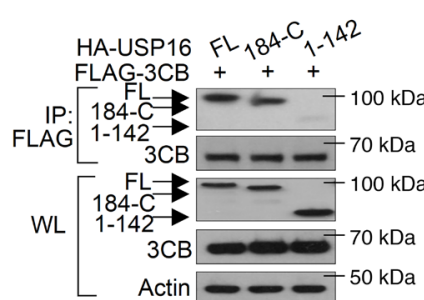

B

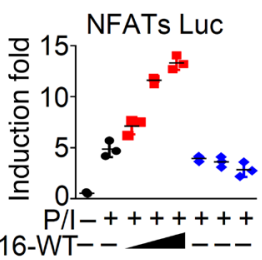

USP16-WT- - - -

USP16-Cl-----

C

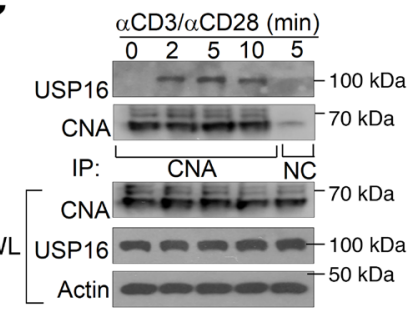

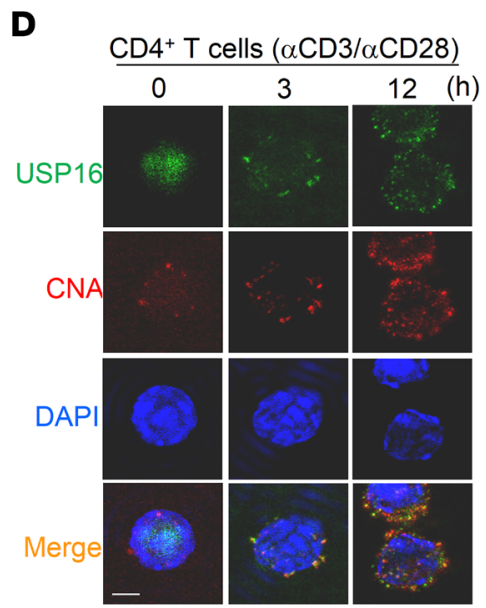

$J$

HA-USP16 - + + FLAG-3CB-W + + FLAG-3CB- $\triangle 86-91-++$
USP16 $-100 \mathrm{kDa}$ FLAG $\left[\begin{array}{r}\text { USP16 } \\ 3 \mathrm{CB} \\ 3 \mathrm{CB} \triangle 86-91\end{array}\right.$ WL $\left[\begin{array}{rr}\text { USP16 } & -100 \mathrm{kDa} \\ 3 \mathrm{CB} & -70 \mathrm{kDa} \\ 3 \mathrm{CB} \triangle 86-91 & \\ \text { Actin } & 50 \mathrm{kDa}\end{array}\right.$

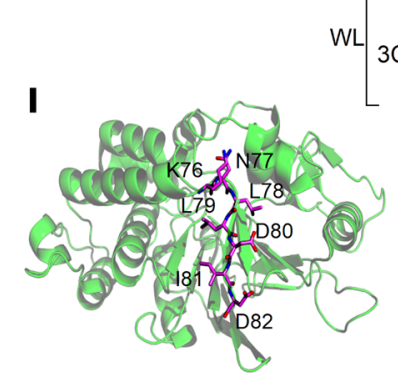

H

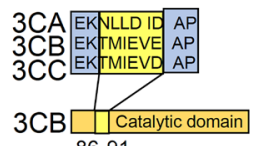
3CB $\underset{8691}{\square}$

\begin{tabular}{|c|c|c|}
\hline $\mathrm{FL} N$ & Catalytic domain & \begin{tabular}{|l|l|l|} 
CBB & CBB & AID \\
\end{tabular} \\
\hline $\mathrm{N}$ & Catalytic domain & \\
\hline $401 \llbracket N$ & Catalytic domain & CBB \\
\hline$4 7 4 \longdiv { N }$ & Catalytic domain & \begin{tabular}{l|l|} 
CBB & CBB \\
\end{tabular} \\
\hline 2-C & Catalytic domain & \begin{tabular}{|l|l|l|l|} 
CBB & CBB & AID \\
\end{tabular} \\
\hline
\end{tabular}

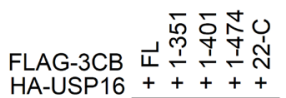
HA-USP16 $++++70 \mathrm{kDa}$ IP: $\left[\begin{array}{r}\text { IB: } \\ \text { FLAG } \\ \text { USP16 }\end{array}\right.$
WL $\left[\begin{array}{r}\text { IB: } \\ \text { FLAG } \\ \text { USP16 } \\ \text { Actin }\end{array}-70 \mathrm{kDa}\right.$
$-50 \mathrm{kDa}$

Figure 2. USP16 selectively associates with CNA encoded by PPP3CB and PPP3CC. (A) HEK293T cells were transfected with 3CB and distinct HA-tagged deubiquitinase (DUB) expression plasmids. IB of 3CB was followed by IP with anti-HA antibody in whole-cell lysates (WLs). (B) WT CD4 ${ }^{+}$T cells were transfected with NFAT luciferase reporters along with WT USP16 (USP16-WT) or its catalytically inactive mutant (USP16-CI) expression plasmids using nucleofection. The P/I-induced readouts were normalized to Renilla luciferase activity and are presented as fold change relative to unstimulated T cells. (C) WT CD4 ${ }^{+}$T cells were stimulated with $\alpha$ CD3/ $\alpha$ CD28, and WLs were subjected to IP using anti-CNA antibody or rabbit IgC control (NC), followed by IB analysis of USP16. The cell lysates were also subjected to direct IB analyses (bottom 3 panels). Immunoprecipitated CNA was detected by Trueblot. (D) Confocal microscopy analysis of the colocalization of USP16, CNA, and DAPI in WT CD4+ T cells stimulated with $\alpha$ CD3/ $\alpha$ CD28 as indicated. Scale bar: $5 \mu$ m. (E) HEK293T cells were transfected with indicated plasmids. IB of FLAG was performed followed by IP with anti-HA antibody in WLs. (F-G) Full-length and various truncated mutants of USP16 (F) or 3CB (G) expression plasmids were cotransfected in HEK293T cells. The interaction of these molecules was detected through the indicated IP and IB analyses. (H) Comparison of the amino acid sequences of the potential USP16-interacting motifs of 3 distinct CNA subunits. (I) Crystal structure of the CNA motif responsible for its interaction with USP16. (J) Full-length CNA and a USP16-interacting defective mutant (3CB- $\triangle 86-91$ ) were coexpressed with USP16 in HEK293T cells. IB of USP16 was performed followed by IP with anti-FLAG antibody in WLs. Data are representative of 3 independent experiments with 3 mice in each group (C-D) and 3 experiments (A, B, E-J).

USP16 specifically removes the K29-linked polyubiquitin chain of CNA. To characterize the DUB activity of USP16, we analyzed the ubiquitination level of CNA after cotransfection with USP16 in HEK293T cells. The results indicated that USP16-WT, but not USP16-CI, could deubiquitinate 3CB and 3CC (Figure 3, A and $B)$. Due to the lack of interaction, USP16 did not promote the deubiquitination of 3CA (Figure 3C). In vitro deubiquitination assays also demonstrated that the USP16-mediated deubiquitination of $3 \mathrm{CB}$ required its DUB activity, because the USP16-CI mutant failed to deubiquitinate 3CB (Figure 3D). We further examined which subtypes of ubiquitin chains were conjugated to CNA and regulated by USP16 in HEK293T cells. As shown, USP16 selectively removed K29-linked polyubiquitin chains from 3CB, and conversely increased K33- and K63-linked ubiquitination (Figure $3 \mathrm{E})$. To confirm the specificity of ubiquitin types, we transfected HA-tagged K29- or K29R-linked ubiquitin expression constructs into WT CD4 ${ }^{+} \mathrm{T}$ cells. Ubiquitination assay revealed that CNA was mainly modified by K29-linked ubiquitin, but not K29R mutant 


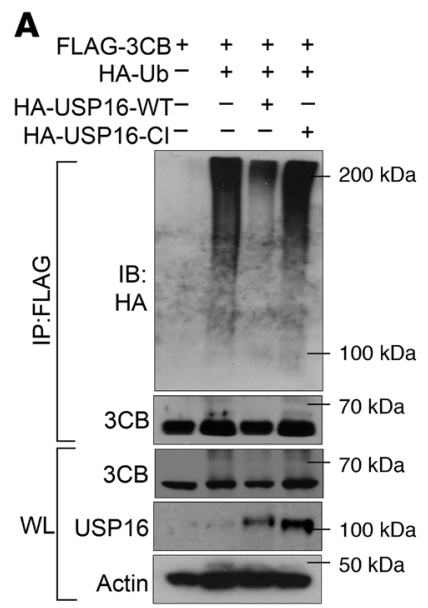

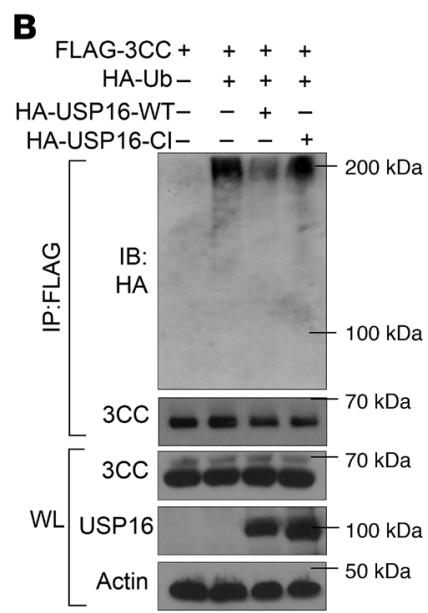

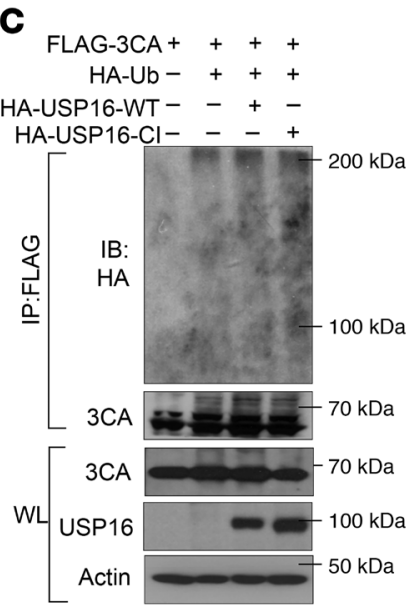

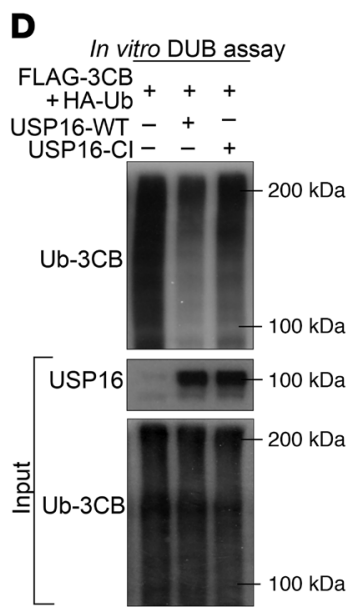

$\mathbf{E}$

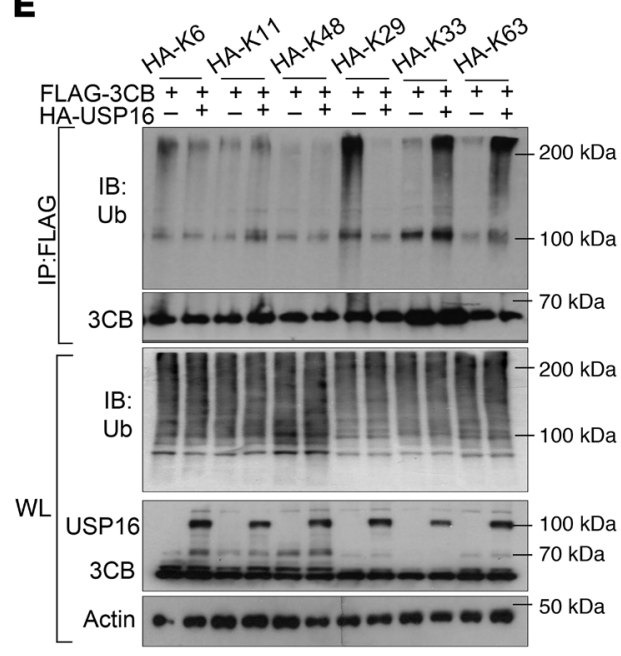

$\mathbf{F}$

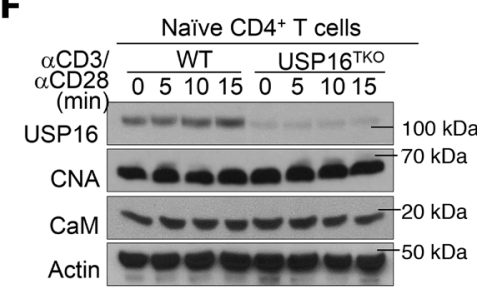

G

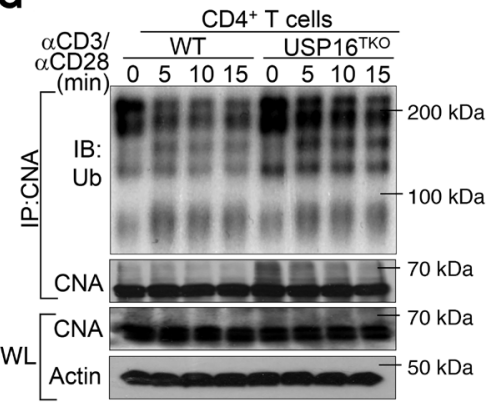

H

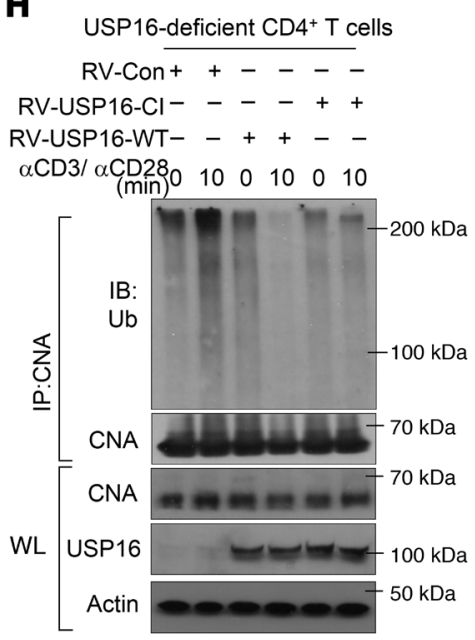

Figure 3. USP16 promotes the deubiquitination of CNA. (A-C) HEK293T cells were transfected with HA-tagged ubiquitin along with the HA-tagged USP16-WT or USP16-Cl expression plasmids. 3CA, ЗСB, and 3CC were isolated by IP with anti-FLAC antibody under denaturing conditions, and followed by the detection of ubiquitin levels with anti-HA. The cell lysates were also subjected to direct IB analyses (bottom 3 panels). (D) In vitro deubiquitination assays were performed to detect the USP16-mediated deubiquitination of 3CB. In vitro translated WT or catalytically inactive USP16 was mixed with ubiquitinated 3CB, which was isolated from transfected HEK293T cells. Deubiquitination of 3CB was detected by direct IB of HA-tagged ubiquitin. (E) HEK293T cells were transfected with multiple HA-tagged ubiquitin mutants, including K6, K11, K48, K63, K29 and K33, along with the indicated expression plasmids. FLAG-tagged 3CB was isolated by IP, and followed by IB detection of ubiquitin. (F) IB analyses of the indicated proteins in WL of CD4 ${ }^{+} \mathrm{T}$ cells from WT and USP16-KO mice stimulated with $\alpha$ CD3/ $\alpha$ CD28. (G) IB analyses of TCR-triggered deubiquitination of CNA in WT and USP16-deficient CD4 ${ }^{+} T$ cells. CNA was isolated by IP and followed by IB detection of ubiquitin. (H) USP16-deficient CD4 ${ }^{+}$T cells were reconstituted with USP16-WT or USP16-CI using nucleofection. After stimulation with $\alpha \mathrm{CD} 3 / \alpha \mathrm{CD} 28$ as indicated, CNA in these reconstituted T cells was isolated by IP and followed by IB detection of ubiquitin. Immunoprecipitated CNA in panels $\mathbf{G}$ and $\mathbf{H}$ was detected by Trueblot. Data are representative of 3 independent experiments with 3 mice in each group (F-H) and $\mathbf{3}$ independent experiments $(\mathbf{A}-\mathbf{E})$.

ubiquitin in resting $\mathrm{CD} 4^{+} \mathrm{T}$ cells (Supplemental Figure 2G). These additional data support our conclusion that inactive CNA participates in K29-linked ubiquitination, which could be removed by USP16 in T cells.

To further confirm the critical role of USP16 in the deubiquitination of endogenous CNA, we generated USP16 $6^{\mathrm{H}}$ mice, and crossed them with $C d 4$-Cre mice to delete USP16 in T cells. As expected, IB analyses revealed a defect in USP16 expression in the USP16-KO T cells. Additionally, TCR stimulation did not affect the protein levels of USP16 in WT T cells at an early stage (Fig- ure $3 F$ ). Although no effect was detected on the protein levels of CNA and CaM (Figure 3F), USP16 deficiency partially suppressed the deubiquitination of CNA triggered by TCR stimulation (Figure 3G and Supplemental Figure 2H). As with the observation in HEK293T cells, we did not detect any signal corresponding to K63-linked ubiquitination of endogenous CNA (Supplemental Figure 2I). We then transfected USP16-deficient $\mathrm{CD} 4^{+} \mathrm{T}$ cells with a retroviral vector encoding the WT or catalytically inactive form of USP16. The data indicated that USP16-WT, but not the USP16CI mutant, could promote the deubiquitination of CNA during 
A

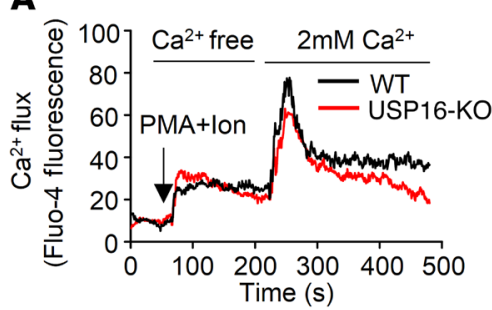

B

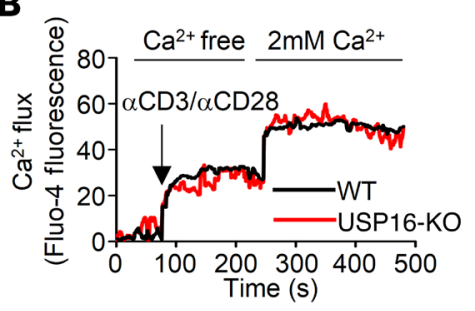

$\mathbf{F}$

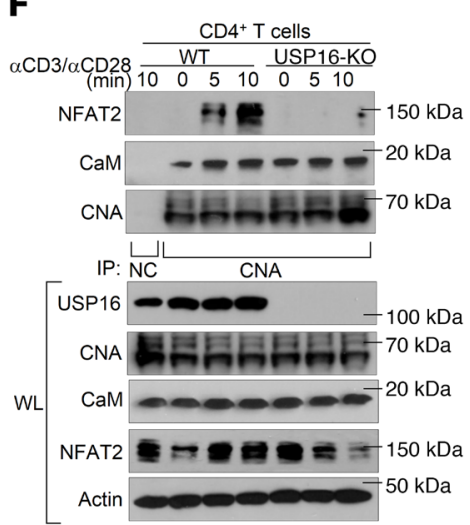

C

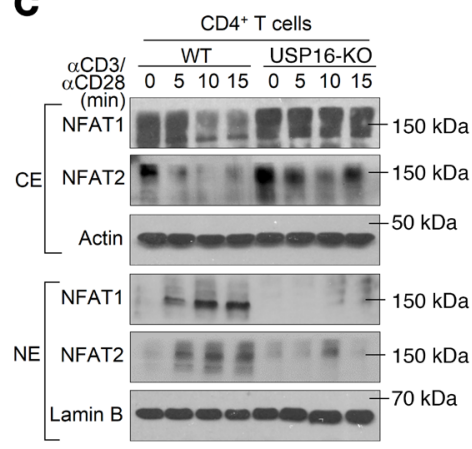

D

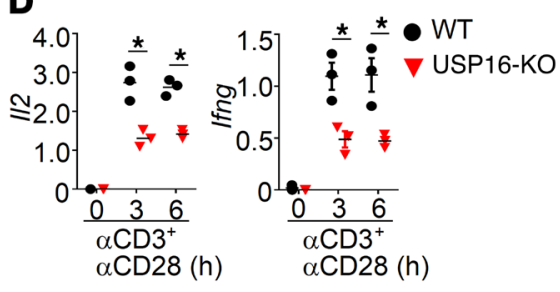

E

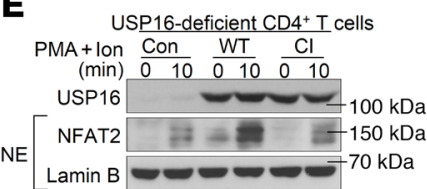

G

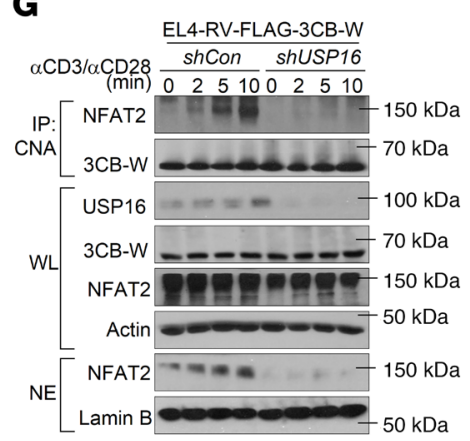

H

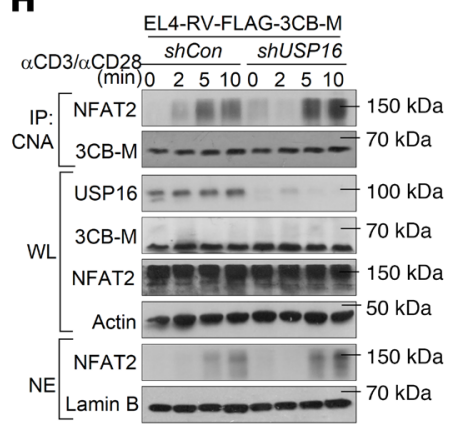

Figure 4. USP16 regulates NFAT activation by controlling its recruitment to CNA. (A and B) Recordings of $\mathrm{Ca}^{2+}$ flux in WT and USP16-deficient CD4+ ${ }^{+}$cells cultured in $\mathrm{Ca}^{2+}$-free medium, and stimulated by crosslinking biotinylated $\mathrm{P} / \mathrm{I}(\mathbf{A})$ or $\alpha \mathrm{CD} 3 / \alpha \mathrm{CD} 28$ (B) along with addition of $2 \mathrm{mM} \mathrm{CaCl}$. (C) IB analysis of the cytoplasmic and nuclear NFAT levels in WT and USP16-deficient CD4+ T cells activated by $\alpha$ CD3/ $\alpha$ CD28. (D) qPCR analysis of $I / 2$ and Ifng mRNA levels in WT or USP16-deficient CD4 ${ }^{+}$T cells in response to plate-coated $\alpha C D 3 / \alpha C D 28$. The qRT-PCR data are presented as fold changes relative to the Actb mRNA level. (E) USP16-deficient CD4+ $T$ cells were reconstituted with USP16-WT or USP16-CI using nucleofection. IB analysis of nuclear translocation of NFAT2 upon P/I stimulation. (F) The interaction between CNA and NFAT2 was assessed in WT and USP16-deficient T cells activated by $\alpha$ CD3/ $\alpha$ CD28. WLs were subjected to IP using anti-CNA antibody or rabbit IgG control (NC), followed by IB analyses of the associated NFAT2. (G-H) EL4 T cells were infected with retrovirus carrying WT (3CB-W) or K327R mutant 3СB (ЗСB-M). GFP+ cells were sorted by FACS, and USP16 expression was silenced by lentiviruses carrying USP16-specific shRNAs. After puromycin selection, these EL4 cells were stimulated with $\alpha$ CD3/ $\alpha$ CD28 as indicated. The nuclear translocation of NFAT2 was evaluated by direct IB analyses. The recruitment of NFAT2 to CNA was assessed in these reconstituted EL4 cells in response to $\alpha$ CD3/ $\alpha$ CD28 stimulation. Immunoprecipitated CNA in F-H was detected by Trueblot. Data are representative of 3 independent experiments with 3 mice each group (A-F) and 3 experiments $(\mathbf{G}$ and $\mathbf{H})$. The error bars show the mean \pm SEM. The significances of differences were determined by 2-tailed Student's $t$ test. Significance was determined by the 2 -tailed Student's $t$ test. ${ }^{*} P<0.05$.

TCR stimulation (Figure 3H). Together these results suggest that USP16 is specifically involved in the K29-linked deubiquitination of $3 \mathrm{CB}$ and $3 \mathrm{CC}$.

USP16-mediated CNA deubiquitination is indispensable for NFAT activation. To clarify the underlying mechanism of USP16-mediated deubiquitination of CNA in activated T cells, we first assessed the P/I- or TCR-induced crucial signaling events. USP16-deficient $\mathrm{CD}^{+} \mathrm{T}$ cells displayed comparable activation levels of MAP kinase phosphorylation (Supplemental Figure 3A) and canonical NF-кB activation (Supplemental Figure 3, B and C). USP16 was also not involved in TCR-proximal signaling transduction, such as phosphorylation of LCK or zeta-chain-associated protein kinase 70 (ZAP70) (Supplemental Figure 3D).
We then evaluated $\mathrm{Ca}^{2+}$ flux in primary $\mathrm{CD}^{+} \mathrm{T}$ cells. FACS analysis indicated that USP16 deficiency did not lead to impairment of the upstream $\mathrm{Ca}^{2+}$ flux (Figure 4, A and B). Consistent with the prior observation in vitro, USP16-deficient $\mathrm{CD} 4^{+} \mathrm{T}$ cells showed reduced activation and nuclear translocation of NFAT1 and NFAT2 stimulated by TCR (Figure 4C and Supplemental Figure $4 \mathrm{~A}$ ) or $\mathrm{P} / \mathrm{I}$ (Supplemental Figure $4, \mathrm{~B}$ and C), which resulted in reduced induction of NFAT-targeted cytokines (Figure 4D). Current evidence indicates that store-operated $\mathrm{Ca}^{2+}$ entry (SOCE) controls the proliferation of T cells through activation of the PI3KAKT kinase-mTOR nutrient-sensing pathway (32). However, USP16 did not impair the phosphorylation of AKT in our study (Supplemental Figure 3D). In reconstituted USP16-deficient 
A

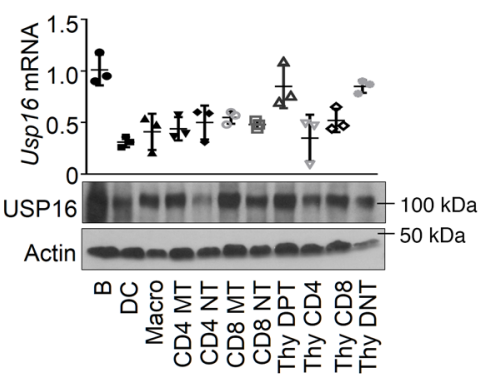

B

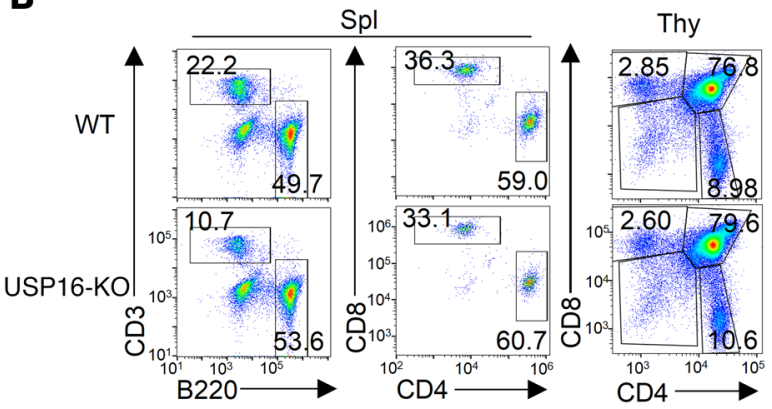

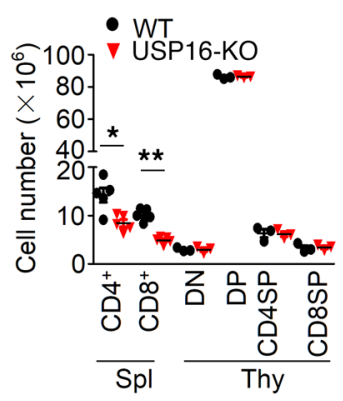

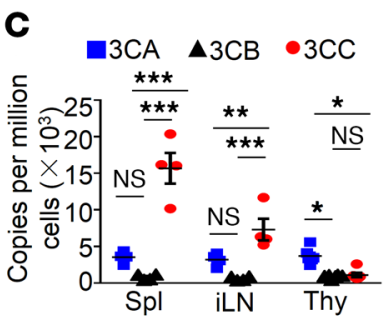

D

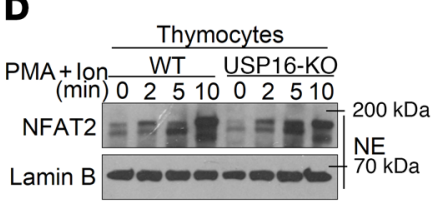

E
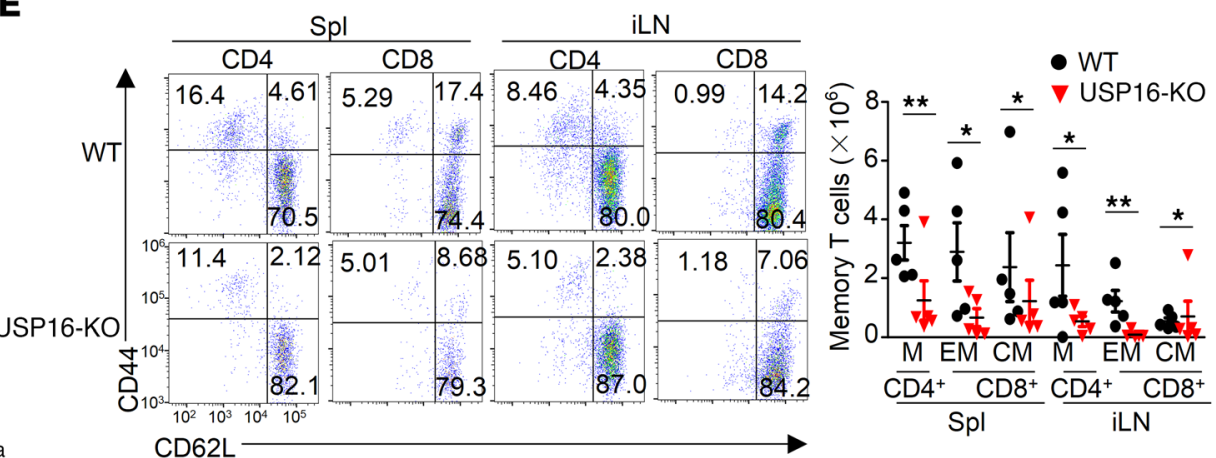

$\mathbf{F}$

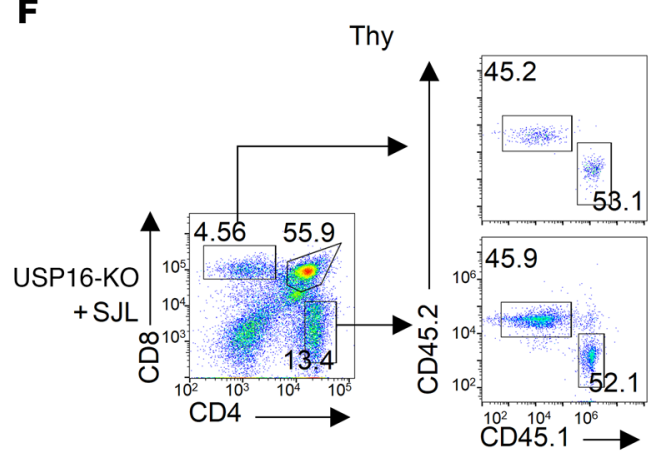

G

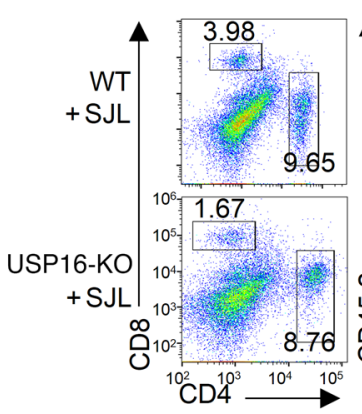

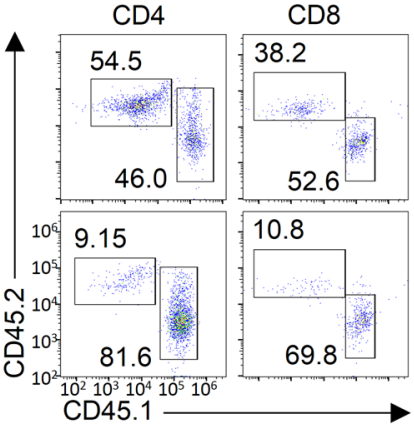

Figure 5. USP16 is indispensable for peripheral T cell maintenance and homeostasis. (A) USP16 expression in various immune cells was monitored by qRT-PCR and IB. (B) Flow cytometric (FACS) analyses of the frequency and absolute cell numbers of subpopulations of cells in the spleen and thymus of 6- to 8-week-old WT and USP16-KO mice. (C) qRT-PCR analyses of the absolute copy numbers of PPP3CA, PPP3CB, and PPP3CC in distinct tissues. The standard curves were generated with different copies of eukaryotic expression plasmids. The same primers were used to measure the expression of 3 CNA isoforms. (D) IB analysis of NFAT2 level in the nucleus of WT and USP16-deficient thymocytes stimulated with P/I as indicated. (E) The cell numbers of T cell subpopulation in the spleen or inguinal lymph nodes (iLNs) from WT and USP16-KO mice evaluated by FACS. (F-G) RAG1-KO recipient mice (6 to 8 weeks old) were adoptively transferred with BM cells derived from 6- to 8-week-old WT or USP16-KO mice (CD45.2 ${ }^{+}$) along with those of B6.SJL mice $\left(C D 45.1^{+}\right)$. The frequency of CD45.1+ or CD45.2+ cells in single-positive population in thymus was measured by FACS (F). The frequency of CD45.1+ or CD45.2 cells in distinct subpopulations of splenic T cells was measured by FACS (G). Data are representative of 3 independent experiments with 3 mice in each group (A, D, F, G) and 4 independent experiments with 6- to 8-week-old mice ( $n=4-5$; female) in each group (B, C, E). The error bars show the mean \pm SEM. The significance of the difference in $\mathbf{C}$ was determined by Dunnett's multiple comparisons test (1-way ANOVA). The significances of differences in 2-group comparisons including B, E, and G were determined by 2-tailed Student's $t$ test. ${ }^{*} P<0.05,{ }^{* *} P<0.01,{ }^{* *} P<0.001$.

$\mathrm{CD}^{+} \mathrm{T}$ cells, USP16-WT, but not USP16-CI, rescued the defective activation of NFAT2 (Figure 4E). Collectively, these findings indicate that USP16 is specifically required for the activation of NFATs without affecting other critical signaling cascades.

To further confirm the role of USP16 in regulating NFAT activation, we examined the molecular interaction of endogenous CNA with CaM or NFAT2. As shown in Figure 4F, the USP16-mediated deubiquitination of CNA was dispensable for its interaction with CaM. Conversely, USP16 deficiency completely disrupted the TCR-triggered recruitment of NFAT2 to CNA (Figure 4F). Consistent with TCR stimuli, extrinsic $\mathrm{Ca}^{2+}$ also promoted the USP16-dependent formation of CNA-NFAT2 complex (Supplemental Figure 4D). To clarify the connection of ubiquitination of CNA with NFAT2 activation, we reconstituted murine T cell line EL4 with WT (3CB-WT) or K327R mutant $3 \mathrm{CB}(3 \mathrm{CB}-\mathrm{M})$ expression constructs, and then knocked 
A

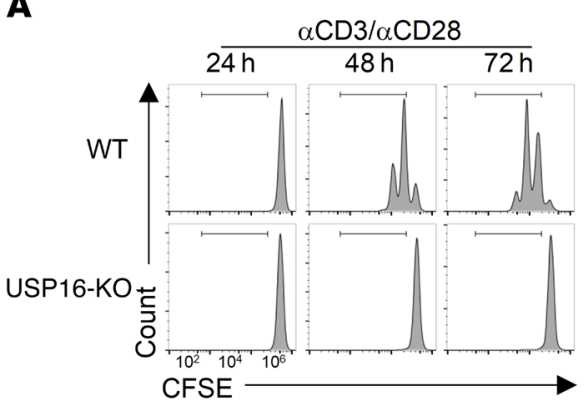

C
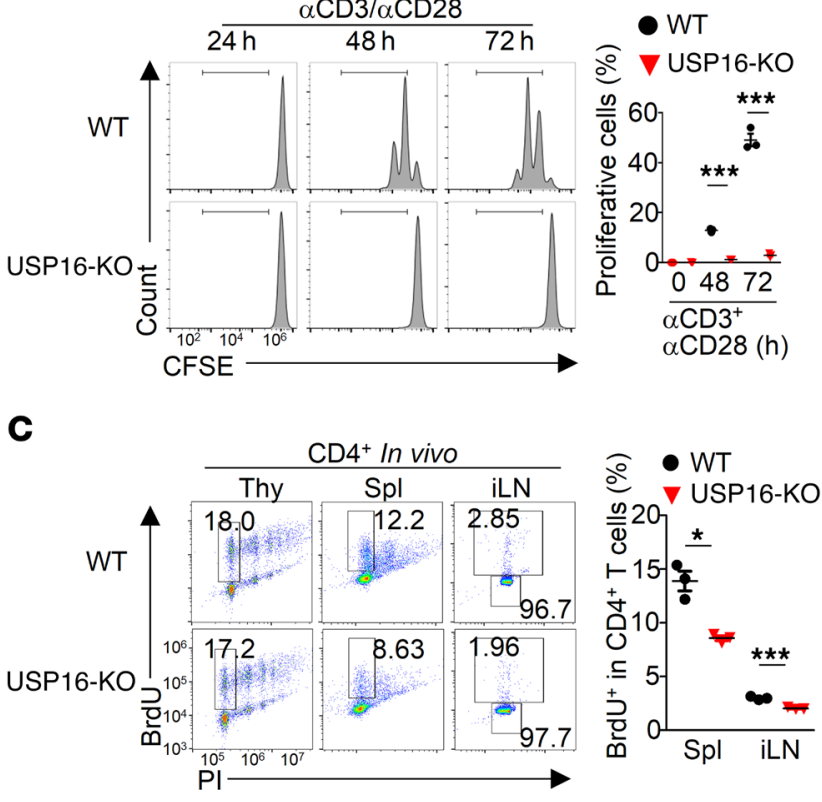

B
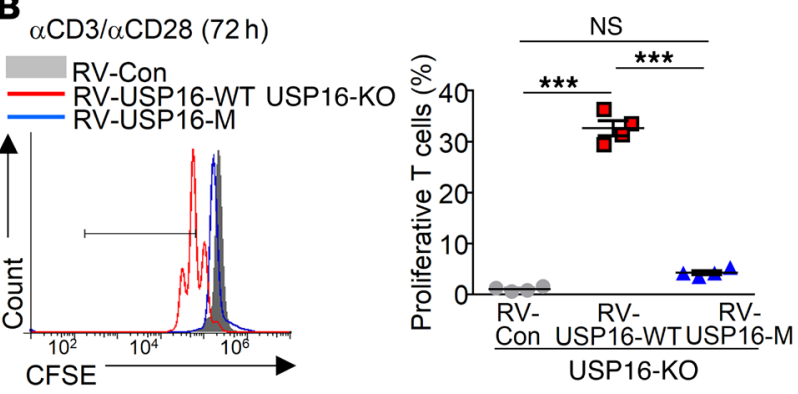

D
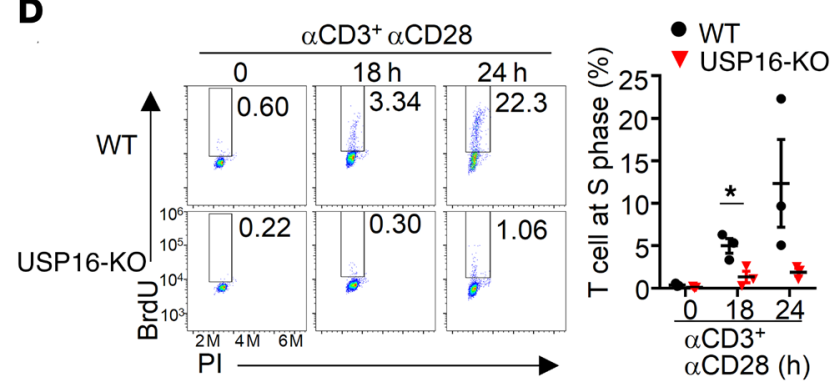

$\mathbf{F}$

$\mathbf{E}$
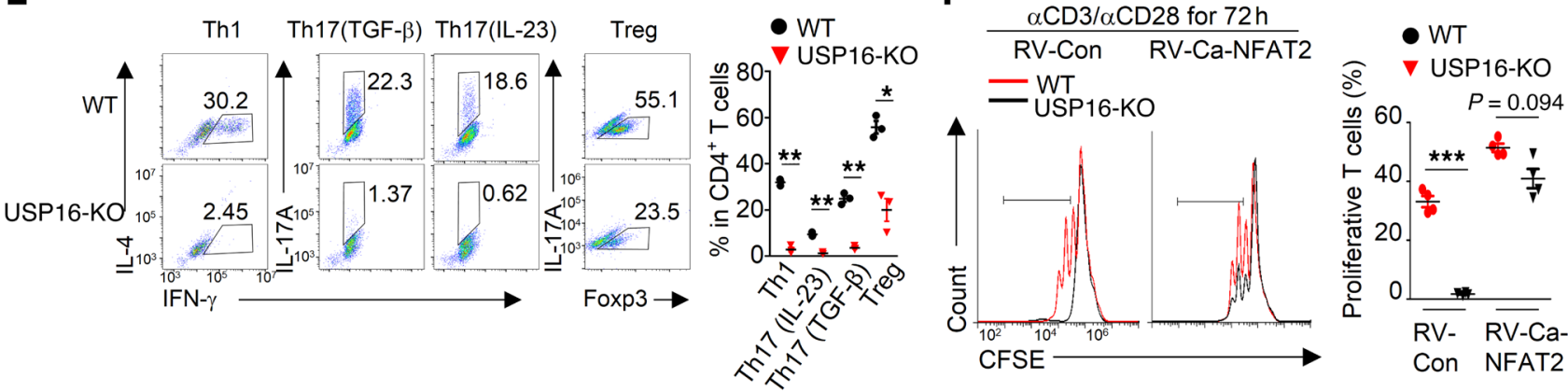

Figure 6. USP16 regulates T cell proliferation and cell-cycle entry. (A) Naive CD4 ${ }^{+}$T cells (CD44 ${ }^{\text {lo }}$ CD62 $L^{\text {hi }}$ ) were isolated from WT and USP16-KO mice by FACS sorter. These T cells were labeled with $5 \mu \mathrm{M}$ CFSE and stimulated with $\alpha \mathrm{CD} 3 / \alpha \mathrm{CD} 28$ as indicated. The proliferative ratio was assessed as CFSE dilution by FACS. (B) USP16-deficient CD4 ${ }^{+}$T cells were infected with retrovirus carrying WT (USP16-WT) or inactive USP16 (USP16-M) for 48 hours, and were selected with puromycin. After resting for 6 hours, the CFSE assay was used for measuring the proliferative capacity of these T cells stimulated with $\alpha$ CD3/ $\alpha$ CD28 for 72 hours. (C and D) Cell-cycle processes and genome replication of naive WT or USP16-deficient CD4+ T cells in vivo (C) and in vitro (D)were evaluated by bromodeoxyuridine (BrdU) staining. (E) Naive CD4+ T cells were isolated from WT and USP16-KO mice, and were stimulated for 4 days with $\alpha$ CD3/ $\alpha$ CD28 under different polarization conditions. The frequency of T cell differentiation was analyzed by FACS based on intracellular staining of the indicated cytokines or specific transcriptional factors. (F) WT or USP16-deficient CD4+ $T$ cells were infected with retrovirus carrying constitutively activated NFAT2 (Ca-NFAT2), and were selected with puromycin. After stimulation with anti-CD3/CD28 for 72 hours, the proliferative capacity of these T cells was measured by CFSE assay. Data are representative of 3 independent experiments with 3 mice in each group (A, C-F) and 4 independent experiments with 4 mice in each group (B). The error bars show the mean \pm SEM. The significance of difference in B was determined by Dunnett's multiple comparisons test. The significances of differences in all other 2 -group comparisons were determined by 2-tailed Student's $t$ test. ${ }^{*} P<0.05$, ${ }^{* *} P<0.01,{ }^{*}{ }^{*} P<0.001$.

down USP16 expression using USP16-specific shRNAs. Similar to that observed in USP16-deficient CD4 ${ }^{+}$T cells, USP16 silencing suppressed the recruitment of NFAT2 to 3CB-WT, but not to 3CB-M (Figure 4, G and H). Thus, USP16 appears to be a critical regulator of CNA activity via the regulation of NFAT recruitment and activation.

USP16 deficiency leads to impaired peripheral $T$ cell maintenance. As an essential regulator of calcineurin, USP16 was found to be mainly distributed in tissues of the immune system, particularly in the spleen and lymph nodes (Supplemental Figure 5A). Analysis of the mRNA and protein levels of USP16 also revealed that USP16 was uniformly expressed in distinct immune cells (Figure 5A). However, USP16-KO mice had consid- erably reduced numbers of $\mathrm{T}$ cells in the spleen and peripheral lymph nodes without changes in the ratio of $\mathrm{CD} 4^{+}$to $\mathrm{CD} 8^{+} \mathrm{T}$ cells (Figure 5B and Supplemental Figure 5, B and C). In addition to reduced numbers of effector $\mathrm{T}$ cells, USP16-KO mice displayed a defect in regulatory $\mathrm{T}$ (Treg) cells (Supplemental Figure 5D). Unexpectedly, FACS analyses indicated comparable frequencies of double-negative (DN) (Supplemental Figure 5E), doublepositive (DP), and single-positive (SP) $\mathrm{CD}^{+}$and $\mathrm{CD}^{+}$thymocytes (Figure $5 \mathrm{~B}$ ), which suggested that USP16 is not involved in $\mathrm{T}$ cell development in the thymus. To clarify the differential regulation of thymocytes and peripheral T cells by USP16, we evaluated the expression levels of $3 \mathrm{CA}, 3 \mathrm{CB}$, and $3 \mathrm{CC}$ in these tissues. qPCR analysis of the absolute copy numbers of these isozymes 
A

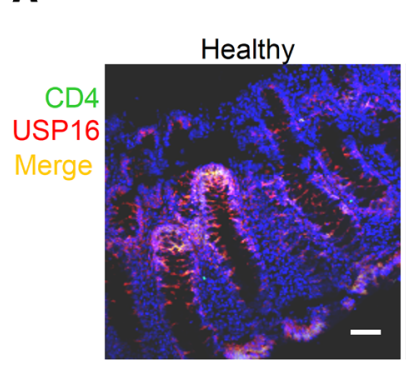

B

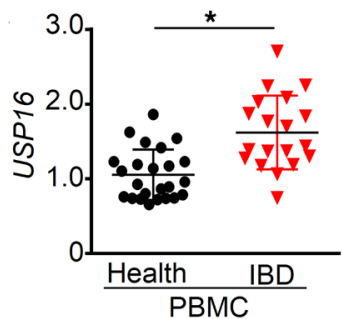

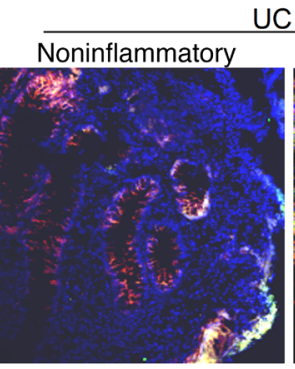

UC
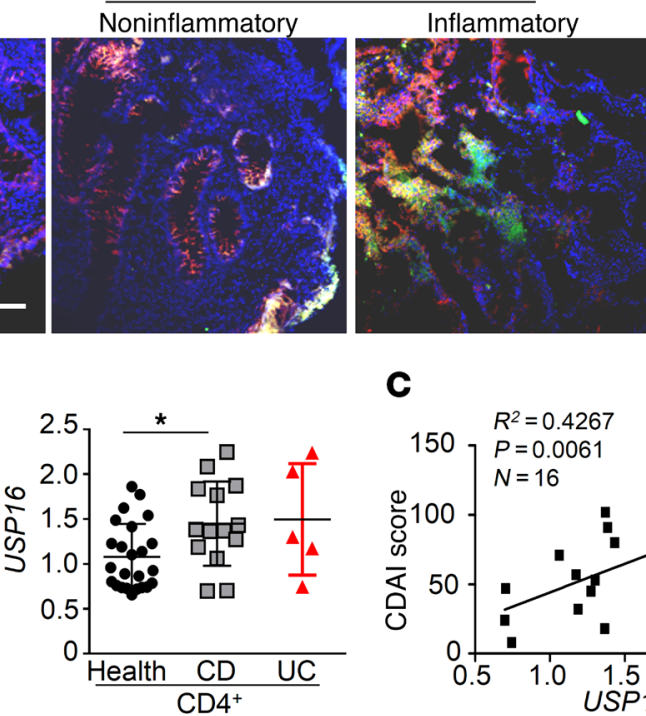

C

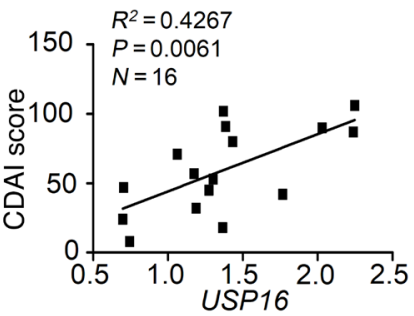

CD

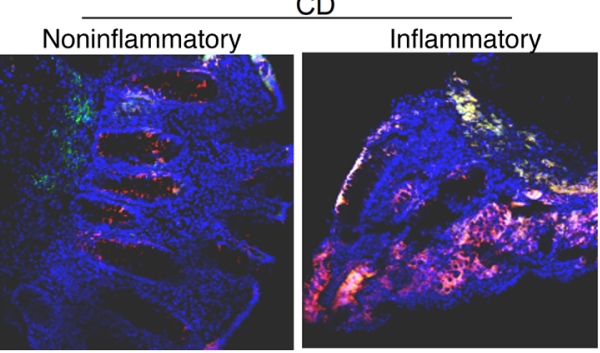

$\mathbf{E}$

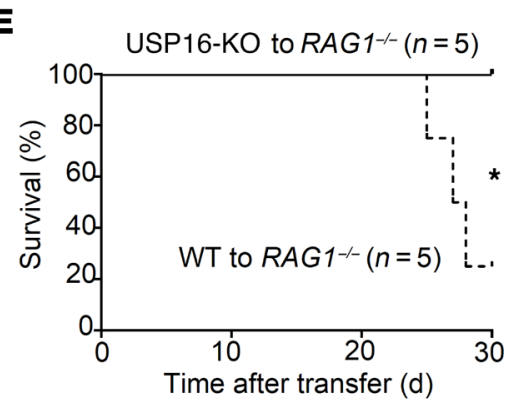

$\mathbf{F}$

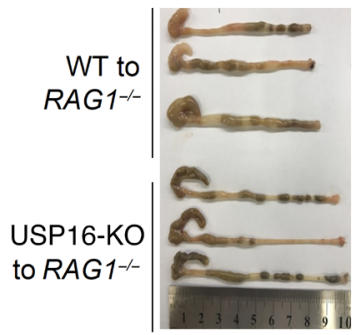

G

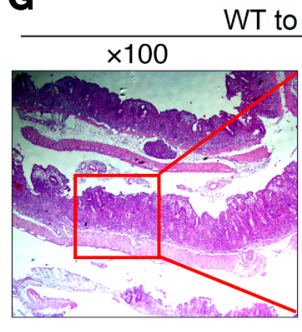

D

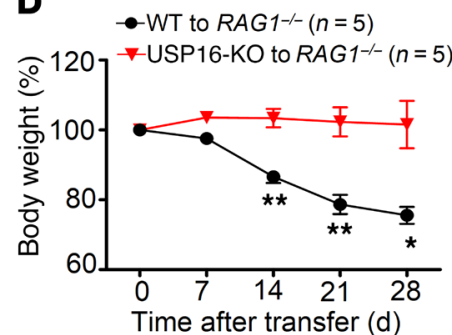

H
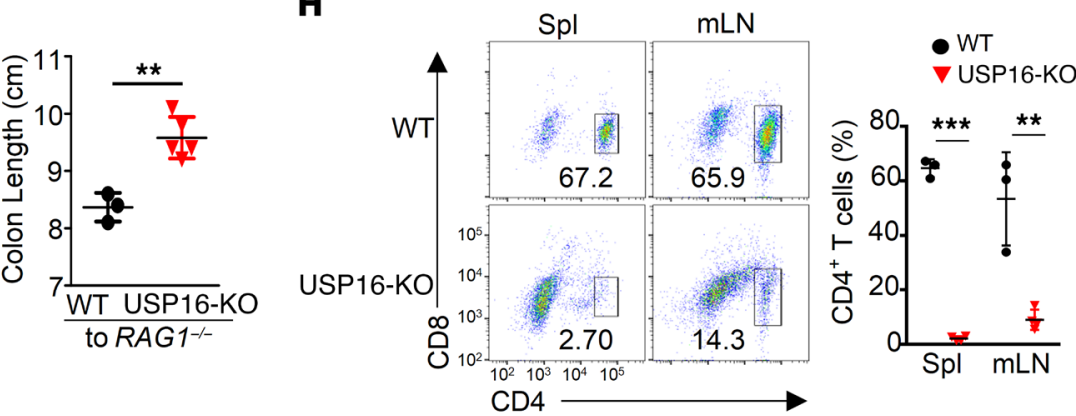

Figure 7. USP16 deficiency alleviates T cell-mediated colitis. (A) Immunofluorescence images of USP16 and CD4 staining in human colon tissue sections from healthy donors or from patients with ulcerative colitis (UC) or Crohn's disease (CD) ( $n=5)$. Scale bar: $50 \mu \mathrm{m}$. (B) PBMCs or CD4+ T cells in peripheral blood from patients with UC or CD, or healthy donors were collected. qRT-PCR analysis of USP16 mRNA levels in these cells. (C) Spearman correlation analysis of USP16 mRNA levels and CD activity index (CDAI) scores. (D-E) Rag1-- mice (6 to 8 weeks old) were intraperitoneally injected with CD4+CD25 CD45RB hi T cells $\left(5 \times 10^{5}\right)$ isolated from 6- to 8-week-old WT or USP16-KO donor mice (female, $n=5 /$ group). Body weight (D) and survival curve (E) were monitored weekly. (F) Four weeks after the injection, the colon length was measured and presented as a summary graph. (C) Representative H\&E slides of proximal colon sections. Scale bar: $200 \mu \mathrm{m}$. (H) FACS analysis of CD4+ $\mathrm{T}$ cells in spleen and mesenteric lymph nodes (mLNs) of the recipient mice. Data are representative of 3 independent experiments with 3 different patients (A), 1 experiment with 5 mice each group ( $\mathbf{D}$ and $\mathbf{E}$ ), and 3 independent experiments with 3 mice each group $(\mathbf{F}$ and $\mathbf{H})$. The error bars show the mean \pm SEM. The significance of difference in $\mathbf{B}$ was determined by Dunnett's multiple comparisons test. Gehan-Breslow-Wilcoxon test was used for $\mathbf{E}$. The significances of differences in all other 2-group comparisons were determined by 2-tailed Student's $t$ test. ${ }^{*} P<0.05 ;{ }^{* *} P<0.01 ;{ }^{* *} P<0.001$.

revealed that 3CA is mainly expressed in thymocytes, whereas $3 \mathrm{CC}$ is mostly distributed in peripheral $\mathrm{T}$ cells in the spleen or lymph nodes (Figure 5C). Because 3CA did not bind USP16, USP16-deficient thymocytes exhibited a comparable nuclear level of NFAT2 after P/I stimulation (Figure 5D and Supplemen- tal Figure 5F). These results suggest that USP16 is only required for the maintenance of peripheral $\mathrm{T}$ cells, but not the development of thymocytes.

In addition to dysregulated $\mathrm{T}$ cell maintenance, USP16-KO mice exhibited a significantly reduced frequency of peripheral 
A

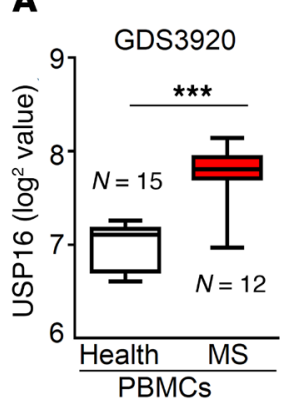

B

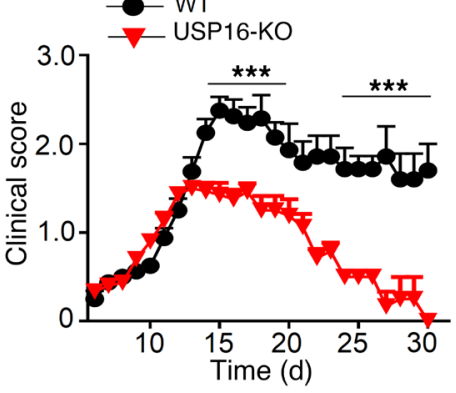

D

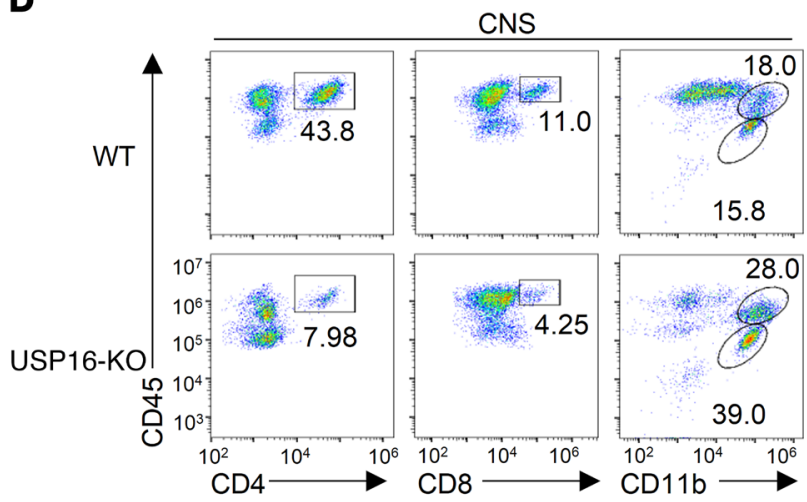

C

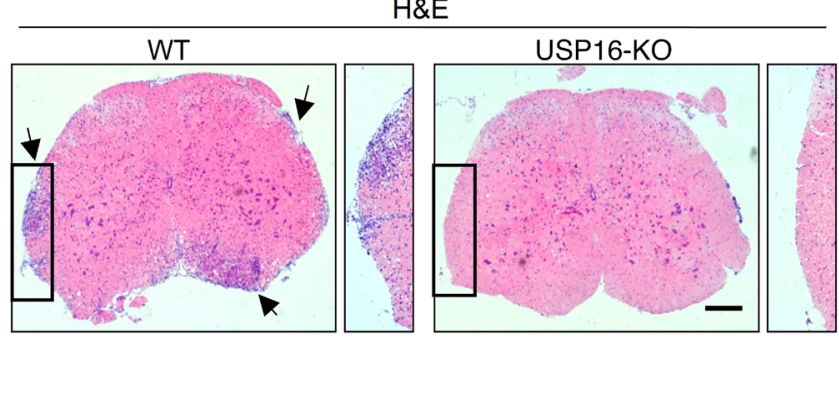

E

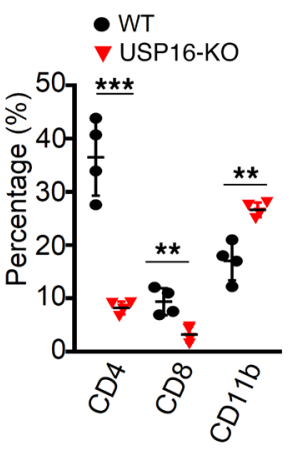

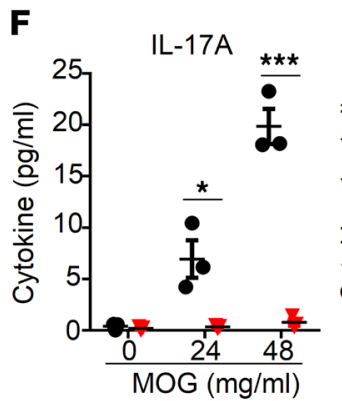

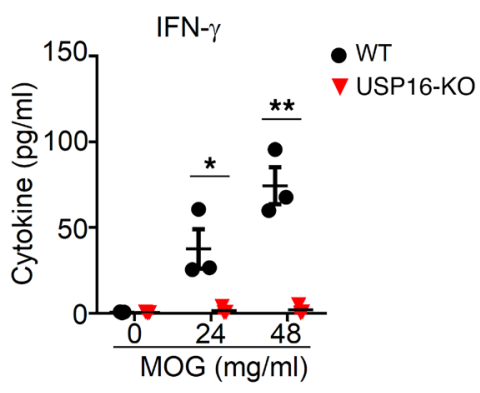

Figure 8. USP16 deficiency in T cells impairs CNS inflammation. (A) Box plot of USP16 expression in multiple sclerosis (MS) patients (using public data set GDS3920; $n=27$ ). (B) Clinical scores of 6- to 8-week-old WT and USP16-KO mice (female, $n=15$ per group) after the induction of experimental autoimmune encephalomyelitis (EAE) with $\mathrm{MOG}_{35-55^{\circ}}$ (C) H\&E staining of spinal cord sections on day 30. Scale bar: $500 \mu \mathrm{m}$. (D) FACS analyses and summary of immune cell infiltration into the brain and spinal cord of mice with $\operatorname{EAE}(n=4$, day 14 postimmunization). (E and $\mathbf{F}$ ) Proliferation and cytokine secretion of splenic $T$ cells from mice with EAE stimulated in vitro with $\mathrm{MOC}_{35-55}(10 \mu \mathrm{g} / \mathrm{ml})$ were measured by CCK8 assay and ELISA, respectively, on day 15. Data are representative of 3 independent experiments with 3 mice in each group (C-F), and 2 experiments with 15 mice each group (B).The error bars show the mean \pm SEM. The significance of difference in $\mathbf{B}$ was determined by Sidak's multiple comparisons test. The significances of differences in all other 2-group comparisons were determined by 2-tailed Student's $t$ test. ${ }^{*} P<0.05,{ }^{* *} P<0.01,{ }^{* *} P<0.001$.

and then cotransferred into RAG1-deficient mice (Supplemental Figure 6A). Six hours later, FACS analysis revealed no significant difference in the proportions of CD $45.1^{+}$and $\mathrm{CD} 45.2^{+} \mathrm{T}$ cells in the lymph nodes or spleen (Supplemental Figure 6B), which indicated that USP16 is dispensable for mature $\mathrm{T}$ cell migration from the thymus to peripheral organs.

It is well established that peripheral $\mathrm{T}$ cells undergo selfrenewal through TCR-mediated cell division. Thus, we assessed the proliferative capacity of naive WT and USP16-deficient CD4 ${ }^{+}$ $\mathrm{T}$ cells stimulated with agonistic antibodies against CD3 and CD28. CFSE-labeled USP16-deficient T cells failed to proliferate, as shown by the lack of reduction in CFSE intensity (Figure 6A). Only WT USP16 restored the proliferative capacity of USP16deficient $\mathrm{CD}^{+} \mathrm{T}$ cells (Figure 6B), suggesting that the catalytic activity of USP16 is essential for T cell proliferation. BrdU incorporation assays indicated that peripheral $\mathrm{T}$ cells from USP16-KO mice were unable to enter the cell cycle both in vivo (Figure 6C) and in vitro (Figure 6D). Similar results were obtained from USP16- 
deficient T cells stimulated with P/I (Supplemental Figure 7, A and B). To exclude a potential function of USP16 in T cell maturation, we crossed USP16 ${ }^{f / f l}$ mice with tamoxifen-inducible Cre $\left(\mathrm{ER}^{\mathrm{Cre}}\right)$ mice to generate inducible-KO USP16 $6^{f / f l} \mathrm{ER}^{\mathrm{Cre}}$ mice. After treatment with 4-hydroxy-tamoxifen (4-OH), the rates of cell division obtained from mature USP16-deficient $\mathrm{T}$ cells were markedly lower than those from WT mice (Supplemental Figure 7C). Consistent with defective proliferation, naive USP16-deficient CD $4^{+} \mathrm{T}$ cells $\left(\mathrm{CD} 44^{\text {lo }} \mathrm{CD} 62 \mathrm{~L}^{\text {hi }} \mathrm{CD} 25^{-}\right.$) were completely unable to polarize into Th1 and Th17 cells in vitro (Figure 6E). Consistently, USP16 deficiency caused impairments in Th2 polarization (Supplemental Figure 7D). Similar to suppressed IL-4 expression, induction of the Th2-specific transcription factor GATA3 was suppressed in USP16-deficient T cells (Supplemental Figure 7E). These data were consistent with previous findings that NFAT1 and NFAT2 are both positive regulators of IL-4 gene transcription $(33,34)$ and indicate that USP16-deficient $\mathrm{T}$ cells might lose their physiological function during the immune response.

Our prior results showed that USP16 regulates T cell proliferation by controlling NFAT activation. Thus, we reconstituted WT and USP16-deficient T cells with a retroviral vector encoding constitutively activated NFAT2 (Ca-NFAT2), which mutates multiple serine residues to alanine residues in the SRR and SPXX repeat motifs. Ca-NFAT2 significantly restored the proliferative capacity of USP16-deficient T cells to their WT control levels (Figure 6F).

USP16 deficiency attenuates $T$ cell-mediated autoimmune diseases. Given the potential importance of USP16 in T cell-mediated inflammation, we first analyzed the expression of USP16 in intestinal tissue collected from healthy individuals or patients with inflammatory bowel disease (IBD). In healthy controls, USP16 is present mainly in intestinal epithelial cells. Conversely, inflammatory conditions triggered an increase in expression of USP16 in colon-infiltrating $\mathrm{CD}^{+} \mathrm{T}$ cells, as demonstrated by a markedly higher fusion signal in the UC and CD sections (Figure 7A). We then assessed the mRNA level of USP16 in PBMCs from patients with autoimmune diseases. As shown in Figure 7B, the mRNA levels of USP16 were clearly higher in patients with IBD than in healthy control individuals. Current evidence suggests a major contribution of T cells to the pathogenesis of $\operatorname{IBD}(35,36)$. To confirm expression of USP16 in pathological T cells, we next examined the mRNA level of USP16 in CD4 ${ }^{+} \mathrm{T}$ cells isolated from the peripheral blood of healthy donors or patients with IBD, including Crohn's disease (CD) and ulcerative colitis (UC). Statistical analysis revealed increased expression of USP16 in CD4 ${ }^{+} \mathrm{T}$ cells from the patients with CD but not UC (Figure 7B). Consistently, patients with higher USP16 mRNA levels in $\mathrm{CD}^{+}{ }^{+} \mathrm{T}$ cells had a significantly higher CD activity index (CDAI) (Figure 7C).

To assess the in vivo function of USP16 in inflammatory diseases, we established a colitis model by transferring effector $\mathrm{T}$ cells. The transfer of naive $\mathrm{CD} 45 \mathrm{RB}^{\text {hi }} \mathrm{CD} 4^{+} \mathrm{T}$ cells from USP16$\mathrm{KO}$ mice into $R A G 1^{-/-}$mice did not induce body weight loss or inflammation-triggered death to the same extent as in WT controls (Figure 7, D and E). Consistently, USP16-deficient T cells did not lead to the shortening of colon length (Figure 7F) or epithelial denudation (Figure 7G). Reduced frequencies of USP16-deficient $\mathrm{T}$ cells were also detected both in spleen and mesenteric lymph nodes (mLNs) (Figure 7H).
To further examine the roles of USP16 in T cell-mediated inflammation, we assessed USP16 expression in other autoimmune diseases, including multiple sclerosis (MS), systemic lupus erythematosus (SLE), and rheumatoid arthritis (RA). qPCR assays and public data sets revealed higher expression of USP16 in $\mathrm{CD}^{+} \mathrm{T}$ cells in patients with MS or SLE than in healthy donors (Figure 8A and Supplemental Figure 8, A and B). Although the mRNA level of USP16 was elevated in $\mathrm{CD}^{+} \mathrm{T}$ cells from patients with RA, no significant increase in USP16 was observed in their synovial tissues (Supplemental Figure 8, C and D). Employing a T cell-dependent autoimmune disease model of experimental autoimmune encephalomyelitis (EAE), we immunized WT and USP16-KO mice with a myelin oligodendrocyte glycoprotein (MOG) peptide $\left(\mathrm{MOG}_{35-55}\right)$ plus pertussis toxin. USP16KO mice exhibited a significantly delayed onset of EAE, demonstrated by the assessment of EAE clinical scores (Figure 8B), and a substantially reduced level of central nervous system infiltration (Figure 8C). In the spinal cord, USP16-KO mice displayed a severe defect in infiltrating $\mathrm{T}$ cells, and a moderate increase in myeloid cells $\left(\mathrm{CD} 11 \mathrm{~b}^{+} \mathrm{CD} 45^{\mathrm{hi}}\right)$ (Figure 8D). Consistently, USP16-deficient $\mathrm{T}$ cells showed a profound defect in recall responses with the MOG peptide, which induced robust proliferation of WT T cells (Figure $8 \mathrm{E})$. Furthermore, the levels of inflammatory cytokines produced by peripheral $\mathrm{T}$ cells from USP16-KO mice with EAE were clearly reduced after stimulation with MOG peptide (Figure 8F). Taken together, these results suggest that USP16 is required for mature $\mathrm{T}$ cell proliferation and $\mathrm{T}$ cell-mediated autoimmune diseases.

\section{Discussion}

Calcium signaling is well established as an essential and ubiquitous cellular signal that regulates various physiological responses in eukaryotes $(2,37)$. Specific signals can open calcium channels in the endoplasmic reticulum (ER) and trigger a rapid increase in $\mathrm{Ca}^{2+}$ levels in the cytoplasm (38-40). The elevated amount of $\mathrm{Ca}^{2+}$ binds with $\mathrm{CaM}$, which alters the activity of calcineurin in the calcium signaling pathway (5). As a critical phosphatase, calcineurin has been identified to interact with many substrates (41). One well-known substrate of calcineurin in activated $\mathrm{T}$ cells is the NFAT family of transcription factors $(3,9)$. NFAT activation is critical for thymocyte development, $\mathrm{T}$ cell activation, differentiation, exhaustion, and self-tolerance (42-44). Cytoplasmic NFATs are constitutively phosphorylated in resting T cells. After dephosphorylation by calcineurin, NFATs translocate to the nucleus and induce the expression of numerous genes, such as IL-2 and IFN- $\gamma$. Calcineurin is also required for the maintenance of NFAT activity in the nucleus upon intracellular $\mathrm{Ca}^{2+}$ stimulation. The established model of calcineurin activation is that elevated intracellular calcium promotes CaM binding to CNA, and induces a conformational change in CNA that leads to the replacement of AID with NFATs $(2,15,37)$. However, many remaining questions regarding the detailed mechanism regulating the activation of calcineurin need to be investigated. One of the major questions is whether the interaction between calcineurin and $\mathrm{CaM}$ is sufficient for its activation. Our findings in the present study suggest that deubiquitination is an essential posttranslational modification of CNA that is indispensable for subsequent NFAT recruitment. Interestingly, this deubiquitination is not required for the CaM bindingtriggered release of the AID. 
In calcium signal-dependent cell types, both protein level and activity of calcineurin are controlled by various ubiquitin ligase complexes. Atrogin-1 associates with S-phase kinaseassociated protein 1 (SKP1), Cullin 1 (CUL1), and RING-box protein 1 (RBX1) to assemble the E3 ubiquitin ligase SCFatrogin-1, which promotes the ubiquitin-dependent proteolysis of CNA in cardiac muscle (45). CNA is also ubiquitinated and degraded by muscle RING-finger 1 (MuRF1), and thereby negatively regulates cardiac hypertrophy in response to pressure overload (PO) $(46,47)$. In addition to CNA, the regulatory subunit of calcineurin $\mathrm{CNB}$ has been found to be degraded by interacting with TNF-receptor associated factor 3 (TRAF3) (48). All of these previous studies focused on the regulation of protein stability, but none has revealed the physiological role of nonproteolytic ubiquitination in calcineurin activity. Here, we demonstrate that CNA in resting T cells exhibits constitutive K29-linked ubiquitination, which can be reversed by USP16. During $\mathrm{T}$ cell activation, USP16 rapidly binds to CNA encoded by $P P P 3 C B$ and PPP3CC. Our results also suggest that modification of 3CA in thymocytes is not regulated by USP16 due to the lack of interaction with USP16. Therefore, USP16 regulates peripheral $\mathrm{T}$ cell maintenance, but is not involved in the positive or negative selection of $\mathrm{T}$ cells in the thymus.

In addition to the role of USP16 in calcium signaling, several important questions emerged from our study, which must be addressed in future studies. First, the E3 ubiquitin ligase that mediates K29-linked ubiquitination of CNA in resting T cells has not yet been identified. Although we clarified the role of USP16 in TCR-triggered CNA deubiquitination through store-operated calcium (SOC) channels, our data in Figure $3 \mathrm{G}$ revealed that the basal ubiquitination level of CNA was higher than that in the WT control. Multiple studies have revealed that resting lymphocytes maintain a low $\mathrm{Ca}^{2+}$ concentration of $100 \pm 7 \mathrm{nmol} / \mathrm{l}$ (49-51). Under resting conditions, $\mathrm{Ca}^{2+}$ influx into $\mathrm{T}$ cells occurs through special constitutively active $\mathrm{Ca}^{2+}$ channels (L-type voltagedependent $\mathrm{Ca}^{2+}$ channels), which are distinct from the receptoractivated SOC channels (52-54). This calcium flux under resting conditions may trigger the partial deubiquitination of CNA in WT T cells, which explains the presence of nonubiquitinated CNA in $\mathrm{CD}^{+} \mathrm{T}$ cells before activation. This flux is also the reason for the high basal level of CNA ubiquitination in USP16deficient $\mathrm{T}$ cells and the defect in peripheral $\mathrm{T}$ cells, even naive $\mathrm{T}$ cells, in USP16-KO mice. These data implied that USP16 might be involved not only in SOC-dependent $\mathrm{Ca}^{2+}$ signal activation but also in calcium signals mediated by voltage-dependent $\mathrm{Ca}^{2+}$ channels in the resting stage. Second, USP16 deficiency induced a weak or moderate impairment in p105 phosphorylation upon TCR stimulation. Thus, the function of USP16 in other signaling pathways, particularly in calcium-insensitive cell types, needs to be further investigated. Third, current evidence indicates that USP16 phosphorylation mediated by some mitosis-related kinases, such as Aurora B or PLK1, enhances its DUB activity $(23,55)$. However, the expression levels of Aurora B and PLK1 were undetectable at the early stages of T cell activation (56). Thus, whether any posttranslational modifications of USP16 are required for its activation and translocation during $\mathrm{T}$ cell activation remains to be investigated. In summary, we elucidated the mechanism by which USP16 regulates the activation of the calcineurin-NFAT axis in calcium signaling, and plays a critical role in peripheral $\mathrm{T}$ cell maintenance. Notably, our findings demonstrate a novel molecular mechanism that might be beneficial for the development of immunosuppressive drugs.

\section{Methods}

Mice and cell lines. USP16 $6^{f / f l}$ mice were purchased from the Model Animal Resource Information Platform, Model Animal Research Center of Nanjing University. The generation of USP1 $6^{f / f l}$ mice was previously described (25). The USP16 ${ }^{f / f l}$ mice were further crossed with Cd4-Cre mice (all from Jackson Laboratory, C57BL/6 background) to generate $\mathrm{T}$ cell-conditional USP16-KO mice (USP16 ${ }^{f / f l} \mathrm{Cd} d$-Cre, USP16-KO). $R A G 1^{-/}, E R^{C r e}$, and SJL mice were from the Model Animal Research Center of Nanjing University. Heterozygous mice were bred to generate littermate controls and conditional $\mathrm{KO}$ mice for experiments. Outcomes of animal experiments were collected blindly and recorded based on ear-tag numbers of the experimental mice. Mice were maintained in specific pathogen-free facility.

The mouse T cell line EL4 and HEK293T cell lines were provided by Shao-cong Sun (MD Anderson Cancer Center, Houston, Texas, USA).

Patients. Clinical biopsies were obtained from inflamed and normal areas of patients with distinct autoimmune diseases and from healthy controls. The samples were immersed in OCT and frozen at $-80^{\circ} \mathrm{C}$. Anticoagulated blood samples $(10 \mathrm{ml})$ were also obtained after overnight fasting from the patients and healthy controls. The blood was used for PBMCs and $\mathrm{CD}^{+} \mathrm{T}$ cell isolation. All patients were from Sir Run Run Shaw Hospital, Zhejiang University School of Medicine (Hangzhou, China). The diagnosis was based on clinical, radiological, and endoscopic examination and histological findings. The severity of diseases was assessed according to international standard criteria such as Crohn's disease activity index (CDAI) for the diagnosis of patients with CD.

Antibodies and reagents. Antibodies against USP16 (B-3), IKB $\alpha$ (C-21), p65 (C-20), Lamin B (C-20), ERK (K-23), phospho-ERK (E-4), JNK (C-17), p38 (H-147), p105/p50 (C-19), AKT1 (B-1), ZAP70 (1E7.2), NFAT2 (7A6), NFAT1 (4G6-G5), CaM (G-3), and Ubiquitin (P4D1) were from Santa Cruz Biotechnology. Antibodies against pancalcineurin A (2614), IKK $\beta$ (L570), BCL10 (C78F1), CARMA1 (1D12), phospho-P65 (Ser536, 93H1), phospho-AKT (Ser473; D9E), phosphoIкB $\alpha$ (Ser32; 9241), phospho-JNK (Thr180/Tyr185; 9251), phospho-p38 (Thr180/Tyr182; 9211), phospho-p105 (Ser933, 18E6), phospho-ZAP70 (Tyr319; 2701), phospho-PLC- $\gamma$ (Tyr783, 2821), and phospho-IKK $\alpha / \beta$ (Ser176/180, 16A6) were purchased from Cell Signaling Technology Inc. Antibodies against calcineurin A encoded by PPP3CA (ab71149) were purchased from Abcam. Anti-Actin (C-4), anti-HA (12AC5), antiFLAG (M2), anti-HA HRP (3F10), and anti-FLAG HRP (M2) were from MilliporeSigma. Fluorescence-labeled antibodies are listed in the section describing flow cytometry and cell sorting.

Plasmids. The retroviral expression vector for HA-tagged human USP16 was provided by Zhang Long (Life Science Institute, Zhejiang University, China). This plasmid was used as a template for creating the catalytically inactive USP16 variant C205S by site-directed mutagenesis. Sequences encoding full-length USP16 isoform 1 and its truncated variants (184-C, 1-142) were subcloned into the HindIII and XbaI sites of the HA-tagged pCDNA vector by PCR. Human PPP3CA, РPР3CB, and $P P P 3 C C$ plasmids in $2 \times F L A G$-tagged PXF2F vector were provided by Zhao Bin (Life Science Institute, Zhejiang 
University, China). $P$ PP $3 C B$-encoded calcineurin and its truncated variants (1-325, 1-351, 1-401, 1-474, 22-C) were subcloned into the $\mathrm{XbaI}$ and BamHI sites of the P3 FLAG-tagged CMV7 vector by PCR. PPP3CB-encoded calcineurin mutants harboring K-to-R substitutions (K327R, K332R, and delta 86-91) were generated by sitedirected mutagenesis using the p3CMV7-FLAG-PPP3CB template and the mutations were confirmed by sequencing. HA-tagged ubiquitin, K11, K29, K33, K48, and K63 plasmids in PRK5 vector and HA-tagged ubiquitin mutants harboring lysine-to-arginine point mutations (K29R) were provided by Shao-cong Sun (MD Anderson Cancer Center, Houston, Texas, USA).

Flow cytometry, cell sorting, and intracellular cytokine staining (ICS). Single cells were subjected to flow cytometry using CytoFlex (Beckman Coulter) and the following fluorescence-labeled antibodies from eBioscience: PB-conjugated anti-CD4; PE-conjugated anti-B220, anti-CD45; PerCP5.5-conjugated anti-Gr-1 (Ly6G), anti-CD45.2 and anti-CD8; APC-conjugated anti-CD3, anti-TCR, and anti-CD62L; APC-CY7-conjugated anti-CD11b and anti-CD45.1; FITC-conjugated anti-CD44, anti-IFN- $\gamma$, and anti-Foxp3.

In spleen and iLNs of normal WT or USP16-KO mice, CD4 ${ }^{+} \mathrm{T}$ cells were defined as total $\mathrm{CD}^{+} \mathrm{CD}^{+}$and $\mathrm{CD}^{+}{ }^{+} \mathrm{T}$ cells as $\mathrm{CD} 3^{+} \mathrm{CD} 8^{+}$. $\mathrm{CD} 4^{+}$ memory (M) T cells were defined as $\mathrm{CD} 4{ }^{+} \mathrm{CD} 44^{\text {hi }} \mathrm{CD} 62 \mathrm{~L}^{\text {lo }} ; \mathrm{CD} 8^{+}$effector memory (EM) $\mathrm{T}$ cells were defined as $\mathrm{CD} 8^{+} \mathrm{CD} 44^{\text {hi }} \mathrm{CD} 62 \mathrm{~L}^{\text {lo }} ; \mathrm{CD} 8^{+}$ central memory $(\mathrm{CM}) \mathrm{T}$ cells were defined as $\mathrm{CD} 8^{+} \mathrm{CD} 44^{\mathrm{hi}} \mathrm{CD} 62 \mathrm{~L}^{\text {hi }}$; and Treg cells were defined as $\mathrm{CD}^{+}{ }^{+} \mathrm{Foxp}^{+}$. In thymus of normal WT or USP16-KO mice, DN cells were defined as $\mathrm{CD} 4^{-} \mathrm{CD} 8$; ; D cells were defined as $\mathrm{CD}^{+}{ }^{+} \mathrm{CD} 8^{+} ; \mathrm{CD} 4^{+} \mathrm{SP}$ cells were defined as $\mathrm{CD} 4^{+} \mathrm{CD} 8^{-}$; and $\mathrm{CD}^{+} \mathrm{SP}$ cells were defined as $\mathrm{CD} 4^{-} \mathrm{CD} 8^{+}$. Among DN thymocytes, group 1 was defined as TCR $\beta^{\text {lo }} \mathrm{CD} 69^{\text {lo }}$; group 2 was defined as TCR $\beta^{\text {mid- }}$ $\mathrm{CD} 69^{\text {lo }}$; group 3 was defined as TCR $\beta^{\text {mid }} \mathrm{CD} 69^{\text {hi }}$; group 4 was defined as TCR $\beta^{\text {hi }} C D 69^{\text {hi }}$; group 5 was defined as TCR $\beta^{\text {hi }}$ CD $69^{\text {mid }}$.

For ICS, T cells were stimulated with PMA $(0.5 \mu \mathrm{g} / \mathrm{ml})$ plus ionomycin $(1 \mu \mathrm{g} / \mathrm{ml})$ for 3 hours and monensin (eBioscience, $\times 1000)$ for another 3 hours, and then subjected to intracellular IFN- $\gamma$ and IL-17A by flow cytometry analysis. Th1 indicates IFN- $\gamma$-expressing $\mathrm{CD} 4^{+} \mathrm{T}$ cells and was defined as CD4+IFN- $\gamma^{+} \mathrm{IL}-4^{-}$; Th17 (TGF- $\beta$ ) indicates TGF- $\beta$-induced IL-17A-expressing $\mathrm{CD}^{+} \mathrm{T}$ cells and was defined as $\mathrm{CD}^{+}{ }^{+}$IL-17A+IFN- $\gamma^{-}$; Th17 (IL-23) indicates IL-23-induced IL-17Aexpressing $\mathrm{CD}^{+}{ }^{+} \mathrm{T}$ cells and was defined as $\mathrm{CD} 4^{+} \mathrm{IL}-17 \mathrm{~A}^{+} \mathrm{IFN}-\gamma^{-}$; and Treg cells were defined as $\mathrm{CD} 4^{+}$Foxp $3^{+} \mathrm{IL}-17 \mathrm{~A}^{-}$.

$\mathrm{T}$ cell isolation and stimulation. Primary $\mathrm{CD} 4^{+} \mathrm{T}$ cells were isolated from the spleen and LNs of young adult mice (6 to 8 weeks old) using anti-CD 4 magnetic beads (Miltenyi Biotec). Naive CD $4^{+}$cells were further purified by flow cytometric cell sorting based on $\mathrm{CD} 4^{+}$ $\mathrm{CD} 44^{\text {lo }} \mathrm{CD} 62 \mathrm{~L}^{\text {hi }}$ surface markers, respectively (Aria II). The cells were stimulated with plate-bound anti-CD3 $(1 \mu \mathrm{g} / \mathrm{ml})$ and anti-CD28 $(1 \mu \mathrm{g} / \mathrm{ml})$ in replicate wells of 96 -well plates $(0.2$ million cells per well) for $\mathrm{T}$ cell proliferation and apoptosis; 48 -well plates ( 0.4 million cells per well) for T cell differentiation; 12 -well plates $\left(10^{6}\right.$ cells per well) for quantitative RT-PCR (qRT-PCR); and 6-well plates $\left(5 \times 10^{6}\right.$ per well) for RNA-Seq assay. Where indicated, the cells were acutely stimulated using an antibody cross-linking protocol.

Bone marrow chimeras. $R A G 1^{-/-}$mice were sublethally irradiated (9.5 Gy) and adoptively transferred with WT (CD45.1 $1^{+}$SJL) mixed with WT and USP16-KO (CD45.2+) bone marrows (1:1) to generate WT and KO chimeric mice, respectively. After 8 weeks, the WT and KO chimeric mice were analyzed by flow cytometry.
$\mathrm{CD}^{+} \mathrm{T}$ cell differentiation. Naive $\mathrm{CD} 4^{+} \mathrm{T}$ cells $\left(\mathrm{CD} 4^{+} \mathrm{CD} 44^{\mathrm{lo}} \mathrm{C}-\right.$ D62 $\mathrm{L}^{\text {hi }}$ ) were isolated from spleens and LNs of WT or USP16-KO mice and stimulated with plate-coated anti-CD3 $(1 \mu \mathrm{g} / \mathrm{ml})$ and anti-CD28 $(1 \mu \mathrm{g} / \mathrm{ml})$ under Th1 (10 ng/ml IL-12 and $5 \mu \mathrm{g} / \mathrm{ml}$ anti-IL-4), Th2 $(10 \mathrm{ng} / \mathrm{ml}$ IL-4, $25 \mathrm{U} / \mathrm{ml} \mathrm{IL-2} \mathrm{and} 5 \mu \mathrm{g} / \mathrm{ml}$ anti-IFN- $\gamma$ ), TGF- $\beta$ Th17 ( $1 \mathrm{ng} / \mathrm{ml}$ TGF- $\beta$ $10 \mathrm{ng} / \mathrm{ml} \mathrm{IL-6,} 5 \mu \mathrm{g} / \mathrm{ml}$ anti-IFN- $\gamma$, and $5 \mu \mathrm{g} / \mathrm{ml}$ anti-IL-4), IL-1 $\beta$ Th17 (10 ng/ml IL-1 $13,10 \mathrm{ng} / \mathrm{ml} \mathrm{IL-6,} 20 \mathrm{ng} / \mathrm{ml} \mathrm{IL}-23,5 \mu \mathrm{g} / \mathrm{ml}$ anti-IFN- $\gamma$, and $5 \mu \mathrm{g} / \mathrm{ml}$ anti-IL-4), or Treg cell ( $1 \mathrm{ng} / \mathrm{ml} \mathrm{TGF}-\beta$ and $10 \mathrm{ng} / \mathrm{ml} \mathrm{IL-2}$ ) conditions. After 4 days of differentiation, cells were subjected to intracellular cytokine staining (ICS) and flow cytometry analyses.

$\mathrm{CD}^{+} \mathrm{T}$ cell proliferation. Naive $\mathrm{CD} 4^{+} \mathrm{T}$ cells were isolated from spleens and LNs of WT or USP16-KO mice. For CFSE dilution assay, $\mathrm{T}$ cells were labelled in $5 \mu \mathrm{M}$ CFSE (Life Technologies) in $37^{\circ} \mathrm{C}$ and washed with PBS 3 times. The cells were normalized and cultured in 96-well plates with plate-coated anti-CD3 $(1 \mu \mathrm{g} / \mathrm{ml})$ and anti-CD28 $(1 \mu \mathrm{g} / \mathrm{ml})$ or with PMA $(0.5 \mu \mathrm{g} / \mathrm{ml})$ and ionomycin $(0.5 \mu \mathrm{g} / \mathrm{ml})$ for 48 and 72 hours. For BrdU incorporation assay, sorted T cells were cultured with $10 \mathrm{mM} \mathrm{BrdU}$ for 18 and 24 hours prior to harvest, stained with BrdU staining kit as per manufacturer's protocol (BD Pharmingen, 559619), and analyzed by flow cytometry. For the in vivo BrdU assay, $120 \mu \mathrm{l}$ of $10 \mathrm{mg} / \mathrm{ml}$ BrdU was intraperitoneally injected into the WT and USP16-KO mice. Twenty-four hours later, the mice were sacrificed and the thymus, spleen, and lymph node cells were stained with BrdU staining kit.

T cell homing. CD4 ${ }^{+} \mathrm{T}$ cells from WT (CD45.1 $\left.1^{+} \mathrm{SJL}\right)$ and USP16-KO $\left(\mathrm{CD} 45.2^{+}\right)$mice were stained with $5 \mu \mathrm{M} \mathrm{CFSE}$ and washed with PBS 3 times. The T cells $\left(10^{7} /\right.$ mouse) were mixed 1:1 and given by tail intravenous injection to $R A G 1^{-/-}$mice, which were lethally irradiated (9.5 Gy). After 6 hours, the recipient mice were sacrificed and the spleen and lymph node cells were stained with CD 45.1 and CD 45.2 and analyzed by flow cytometry.

Induction and assessment of EAE. For active EAE induction, age- and sex-matched mice were immunized s.c. with $\mathrm{MOG}_{35-55}$ peptide $(300 \mu \mathrm{g})$ mixed in CFA (Sigma-Aldrich) containing $5 \mathrm{mg} / \mathrm{ml}$ heat-killed Mycobacterium tuberculosis H37Ra (Difco). Pertussis toxin (200 ng, List Biological Laboratories) in PBS was administered i.v. on days 0 and 2. Mice were examined daily and scored for disease severity using the standard scale: 0 , no clinical signs; 1 , limp tail; 2, paraparesis (weakness, incomplete paralysis of one or 2 hind limbs); 3 , paraplegia (complete paralysis of 2 hind limbs); 4, paraplegia with forelimb weakness or paralysis; 5 , moribund or death. After the onset of EAE, food and water were provided on the cage floor. Mononuclear cells were prepared from the CNS (brain and spinal cord) of EAE-induced mice and analyzed by flow cytometry. The CNS-infiltrating $\mathrm{CD} 4^{+} \mathrm{T}$ cell was defined as $\mathrm{CD} 4^{+} \mathrm{CD} 45^{\text {hi }}$; the CNSinfiltrating $\mathrm{CD}^{+} \mathrm{T}$ cell was defined as $\mathrm{CD} 8^{+} \mathrm{CD} 45^{\text {hi }}$; the CNS-infiltrating myeloid cell was defined as $\mathrm{CD} 11 \mathrm{~b}^{+} \mathrm{CD} 45^{\text {hi }}$; the resident microglia cell was defined as $\mathrm{CD} 11 \mathrm{~b}{ }^{+} \mathrm{CD} 45^{\mathrm{mid}}$.

$T$ cell adoptive transfer model of colitis. $\mathrm{CD} 4^{+} \mathrm{CD} 25^{-} \mathrm{CD} 45 \mathrm{RB}{ }^{\text {hi }}$ cells from WT and USP16-KO mice were prepared by FACS sorting and adoptively transferred (via i.v. injection) into $R A G 1^{-/-}$mice $\left(5 \times 10^{5}\right.$ cells/mouse). Recipient mice were observed daily, and body weight was measured weekly. At the end of the experiment (4 weeks), all mice were sacrificed and intestines were removed for flow cytometry analysis, H\&E staining, and histology analysis.

ELISA and $q R T-P C R$. Supernatants of in vitro cell cultures were analyzed by ELISA using a commercial assay system (eBioScience). For qRT-PCR, total RNA was isolated using TRI reagent (Molecular 
Research Center, Inc.) and subjected to cDNA synthesis using RNase H-reverse transcriptase (Invitrogen) and oligo (dT) primers. qRT-PCR was performed in triplicate, using iCycler Sequence Detection System (Bio-Rad) and iQTM SYBR Green Supermix (Bio-Rad). The expression of individual genes was calculated by a standard curve method and normalized to the expression of Actb. The gene-specific PCR primers (all for mouse genes) are shown in Supplemental Table 1.

Preparation of nuclear versus cytosolic fractions. Cells were lysed in buffer B lysis buffer (10 mM Hepes pH 7.9, $10 \mathrm{mM} \mathrm{KCl,} 0.1 \mathrm{mM}$ EDTA, 0.4\% NP-40, 1 mM DTT, 0.1 mM PMSF), and left on ice for 15 minutes. After spinning (12,000 rpm) for 15 seconds, the supernatant was transferred into a new tube (cytosol fraction). The nuclear pellets were washed with $500 \mu \mathrm{l}$ buffer B 3 times. After removing the washing buffer, buffer C (20 mM Hepes pH 7.9, 0.4 mM NaCl, 1 mM EDTA, 1 mM DTT, $1 \mathrm{mM}$ PMSF) was added to the nuclear pellets. These pellets were shaken vigorously for 10 minutes on a vortex shaker $\left(4^{\circ} \mathrm{C}\right)$. Following centrifugation $(12,000 \mathrm{rpm})$ for 1 minute, the supernatants were collected and transferred into a new tube (nuclear extract). $\beta$-actin and Lamin B were used as loading controls for the cytoplasm and nucleus, respectively.

$I B, I P$, and kinase assays. Whole-cell lysates or subcellular extracts were prepared and subjected to IB and IP assays as previously described (57). In immunoprecipitated (IP) experiments, we pulled down calcineurin A in all primary $\mathrm{T}$ cells or the $\mathrm{T}$ cell line (EL4) by using pancalcineurin A antibody (anti-CNA). In transfected 293T cells, we used FLAG antibody to immunoprecipitate the tagged CNA (3CA, 3CB, or 3CC), as indicated in the Figures. The samples were resolved by $8.25 \%$ SDS-PAGE. After electrophoresis, separated proteins were transferred onto PVDF membrane (Millipore). For immunoblotting, the PVDF membrane was blocked with 5\% nonfat milk. After incubation with specific primary antibody, horseradish peroxidase-conjugated secondary antibody was applied. The positive immune reactive signal was detected by ECL (Amersham Biosciences). See complete unedited blots in the supplemental material.

Ubiquitination assays. Cells were lysed with a Nonidet $\mathrm{P}-40$ lysis buffer (50 mM Tris-HCl, pH 7.5, $120 \mathrm{mM} \mathrm{NaCl}, 1 \%$ Nonidet P-40, $1 \mathrm{mM}$ EDTA, and $1 \mathrm{mM}$ DTT) containing $6 \mathrm{M}$ urea and protease inhibitors. This lysis was subsequently diluted with NP-40 lysis buffer before immunoprecipitation. Pan-calcineurin or FLAG was isolated by IP with anti-calcineurin or anti-FLAG. The ubiquitinated proteins were detected by IB using an antiubiquitin (Santa Cruz Biotechnology, P4D1) antibody.

In vitro transcription-coupled translations. USP16 protein and its USP16C205S mutant were synthesized with pcDNA3-HA-USP16 as a template in rabbit reticulocyte lysates, and programmed with the corresponding RNA using transcription-coupled translations (TNTs) in vitro translation kits (Promega). NFAT2 and the AID domain of CNA were produced similarly based on the protocol from the kit described as above. The gene-specific PCR primers are shown in Supplemental Table 2.

LC-MS/MS analysis. The tryptic peptides were dissolved in $0.1 \%$ formic acid (solvent A), directly loaded onto a homemade reversedphase analytical column (15-cm length, $75 \mu \mathrm{m}$ i.d.). The gradient was comprised of an increase from $6 \%$ to $23 \%$ solvent B (0.1\% formic acid in $98 \%$ acetonitrile) over 16 minutes, $23 \%$ to $35 \%$ in 8 minutes, and climbing to $80 \%$ in 3 minutes then holding at $80 \%$ for the last 3 minutes, all at a constant flow rate of $400 \mathrm{nl} / \mathrm{min}$ on an EASY-nLC
1000 UPLC system. The peptides were subjected to NSI source followed by tandem mass spectrometry (MS/MS) in Q Exactive Plus (Thermo) coupled online to the UPLC. The electrospray voltage applied was $2.0 \mathrm{kV}$. The $\mathrm{m} / \mathrm{z}$ scan range was 350 to 1800 for full scan, and intact peptides were detected in the Orbitrap at a resolution of 70,000. Peptides were then selected for MS/MS using the NCE setting as 28 , and the fragments were detected in the Orbitrap at a resolution of 17,500. A data-dependent procedure alternated between 1 MS scan followed by $20 \mathrm{MS} / \mathrm{MS}$ scans with 15.0 s dynamic exclusion. Automatic gain control (AGC) was set at $5 \mathrm{E} 4$.

Confocal microscopy. $\mathrm{CD}^{+} \mathrm{CD} 25^{-} \mathrm{CD} 45 \mathrm{RB}^{\text {hi }}$ cells from WT and USP16-KO mice were collected and seeded on a 12-well plate without or with plate-coated anti-CD3 $(1 \mu \mathrm{g} / \mathrm{ml})$ and anti-CD28 $(1 \mu \mathrm{g} / \mathrm{ml})$ for 3 to 12 hours. Cells were fixed in prewarmed $4 \%$ paraformaldehyde for 30 minutes. After blocking with 5\% BSA, cells were stained overnight with anti-USP16 and anti-calcineurin (1:100). Staining was detected using DyLight 488- and DyLight 549-labelled secondary antibodies (1:1000; Multiscience). Nuclei were costained with DAPI (Roche). All the samples were imaged on a LSM710 (Carl Zeiss) confocal microscope outfitted with a Plan-Apochromat $\times 63$ oil immersion objective lens (Carl Zeiss). The data were collected using Carl Zeiss software ZEN 2010. Colocalization analyses were performed on more than 10 cells was expressed as a Pearson's coefficient (R).

Calcium flux. Thymocytes or naive $\mathrm{CD} 4^{+} \mathrm{T}$ cells from spleen and lymph node in suspensions $\left(3 \times 10^{6}\right)$ were labeled for 1 hour at $37^{\circ} \mathrm{C}$ with 4 $\mathrm{g} / \mathrm{ml}$ Fluo4 (Invitrogen), then were washed with ice-cold PBS and resuspended in PBS. Cells were incubated with biotinylated anti-CD3 $(5 \mu \mathrm{g} /$ $\mathrm{ml}$; 145-2C11; eBioscience) and anti-CD28 (5 $\mu \mathrm{g} / \mathrm{ml}$; 37.51; BioLegend), and warmed for 20 minutes at room temperature before flow cytometry (Cytoflex). After 30 seconds, the cells were crosslinked with streptavidin $(25 \mu \mathrm{g} / \mathrm{ml})$ or were stimulated with PMA (phorbol 12-myristate 13acetate; $0.5 \mu \mathrm{g} / \mathrm{ml})$ and ionomycin $(0.5 \mu \mathrm{g} / \mathrm{ml})$ during the cytometry. $\mathrm{CaCl}_{2}$ was added to the tube 180 seconds later, and the $\mathrm{Ca}^{2+}$ concentration was $2 \mathrm{mM}$. The detection was maintained for 480 seconds. Mean fluorescence ratios were plotted after analysis with FlowJo software (TreeStar).

Statistics. Statistical analysis was performed using Prism software. For 2-group comparison, 2-tailed unpaired $t$ tests were performed. For multiple group comparison, Dunnett's multiple comparison test (1-way ANOVA) was performed. The Gehan-Breslow-Wilcoxon test was used for the animal survival assay. In the EAE model, clinical scores were analyzed by Sidak's multiple comparison test (2-way ANOVA) with $90 \%$ power and a significance level of $5 \%$. All statistical tests are justified as appropriate, and data meet the assumptions of the tests. The groups being statistically compared show similar variance. $P$ values less than 0.05 were considered significant. Levels of significance were indicated as ${ }^{*} P<0.05,{ }^{* *} P<0.01$, and ${ }^{* *} P<0.001$.

Study approval. All animal experiments were conducted in accordance with protocols (no. 12077) approved by the Institutional Animal Care and Use Committee of Zhejiang University. For human studies, written informed consent was obtained from all subjects before the study protocol.

\section{Author contributions}

Y Zhang, RL, QC, and KF conducted experiments and acquired and analyzed data. LH,JY,ZG, TH, JZ, XM, FW, PX, and Y Zhao conducted experiments. XF and YL designed research studies. JJ designed research studies, analyzed data, and wrote the manuscript. 


\section{Acknowledgments}

We thank Lin-rong Lu, Yi-chuan Xiao, Shao-cong Sun, and Bin Zhao for expression plasmid. This study was supported by the Excellent Young Scientists Fund of Natural Science Foundation of China (NSFC) (31822017). This study was also supported by Zhejiang Provincial NSFC under grants LR19C080001 and LQ19H030014, and by the National NSFC (81572651/81771675), Fundamental Research Funds for the Central Universities (2018LZA6015).

Address correspondence to: Jin Jin, A435, Nano Building, Zhejiang University Zijingang Campus, Xihu, Hangzhou, Zhejiang Province, China. Phone: 86.571.88208536; Email:jjin4@zju.edu.cn.
1. Klee CB, Crouch TH, Krinks MH. Calcineurin: a calcium- and calmodulin-binding protein of the nervous system. Proc Natl Acad Sci U S A. 1979;76(12):6270-6273.

2. Clapham DE. Calcium signaling. Cell. 2007;131(6):1047-1058.

3. Crabtree GR, Olson EN. NFAT signaling: choreographing the social lives of cells. Cell. 2002;109 Suppl:S67-S79.

4. Hogan PG, Chen L, Nardone J, Rao A. Transcriptional regulation by calcium, calcineurin, and NFAT. Genes Dev. 2003;17(18):2205-2232.

5. Crabtree GR. Calcium, calcineurin, and the control of transcription. J Biol Chem. 2001;276(4):2313-2316.

6. Mencarelli A, et al. Calcineurin-mediated IL-2 production by CD11c. Nat Commun. 2018;9(1):1102.

7. Dutta D, et al. Recruitment of calcineurin to the TCR positively regulates T cell activation. $\mathrm{Nat}$ Immunol. 2017;18(2):196-204.

8. Alam MS, et al. Unique properties of TCR-activated p38 are necessary for NFAT-dependent T-cell activation. PLoS Biol. 2018;16(1):e2004111.

9. Macian F. NFAT proteins: key regulators of T-cell development and function. Nat Rev Immunol. 2005;5(6):472-484.

10. Nguyen T, Di Giovanni S. NFAT signaling in neural development and axon growth. Int J Dev Neurosci. 2008;26(2):141-145.

11. McCullagh KJ, et al. NFAT is a nerve activity sensor in skeletal muscle and controls activity-dependent myosin switching. Proc Natl Acad Sci US A. 2004;101(29):10590-10595.

12. Schulz RA, Yutzey KE. Calcineurin signaling and NFAT activation in cardiovascular and skeletal muscle development. Dev Biol. 2004;266(1):1-16.

13. Calabria E, et al. NFAT isoforms control activity-dependent muscle fiber type specification. Proc Natl Acad Sci U S A . 2009;106(32):13335-13340.

14. Hashimoto Y, Perrino BA, Soderling TR. Identification of an autoinhibitory domain in calcineurin. J Biol Chem. 1990;265(4):1924-1927.

15. Li SJ, et al. Cooperative autoinhibition and multi-level activation mechanisms of calcineurin. Cell Res. 2016;26(3):336-349.

16. Kingsbury TJ, Cunningham KW. A conserved family of calcineurin regulators. Genes Dev. 2000;14(13):1595-1604.

17. Valdor R, et al. Chaperone-mediated autophagy regulates $\mathrm{T}$ cell responses through targeted degradation of negative regulators of $\mathrm{T}$ cell activation. Nat Immunol. 2014;15(11):1046-1054.

18. De Windt LJ, et al. Targeted inhibition of calcineurin attenuates cardiac hypertrophy in vivo. Proc Natl Acad Sci USA. 2001;98(6):3322-3327.

19. Taigen T, De Windt LJ, Lim HW, Molkentin JD. Targeted inhibition of calcineurin prevents agonist-induced cardiomyocyte hypertrophy.
Proc Natl Acad Sci U S A. 2000;97(3):1196-1201.

20. Li X, Matta SM, Sullivan RD, Bahouth SW. Carvedilol reverses cardiac insufficiency in AKAP5 knockout mice by normalizing the activities of calcineurin and CaMKII. Cardiovasc Res. 2014;104(2):270-279.

21. Kafadar KA, Zhu H, Snyder M, Cyert MS. Negative regulation of calcineurin signaling by Hrr25p, a yeast homolog of casein kinase I. Genes Dev. 2003;17(21):2698-2708.

22. Liu YC, Storm DR. Dephosphorylation of neuromodulin by calcineurin. J Biol Chem . 1989;264(22):12800-12804.

23. Zhuo X, et al. Usp16 regulates kinetochore localization of Plk1 to promote proper chromosome alignment in mitosis. JCell Biol. 2015;210(5):727-735.

24. Gu Y, et al. The histone $\mathrm{H} 2 \mathrm{~A}$ deubiquitinase Usp16 regulates hematopoiesis and hematopoietic stem cell function. Proc Natl Acad Sci U S A. 2016;113(1):E51-E60.

25. Yang W, et al. The histone H2A deubiquitinase Usp16 regulates embryonic stem cell gene expression and lineage commitment. Nat Com mun. 2014;5:3818.

26. Adorno M, et al. Usp16 contributes to somatic stem-cell defects in Down's syndrome. Nature. 2013;501(7467):380-384.

27. Buitenkamp TD, et al. Acute lymphoblastic leukemia in children with Down syndrome: a retrospective analysis from the Ponte di Legno study group. Blood. 2014;123(1):70-77.

28. Qian Y, et al. USP16 downregulation by carboxyl-terminal truncated HBx promotes the growth of hepatocellular carcinoma cells. Sci Rep. 2016;6:33039.

29. Joo HY, et al. Regulation of cell cycle progression and gene expression by H2A deubiquitination. Nature. 2007;449(7165):1068-1072.

30. Mimnaugh EG, et al. Caspase-dependent deubiquitination of monoubiquitinated nucleosomal histone $\mathrm{H} 2 \mathrm{~A}$ induced by diverse apoptogenic stimuli. Cell Death Differ. 2001;8(12):1182-1196.

31. Cai SY, Babbitt RW, Marchesi VT. A mutant deubiquitinating enzyme (Ubp-M) associates with mitotic chromosomes and blocks cell division. Proc Natl Acad Sci U S A. 1999;96(6):2828-2833.

32. Vaeth $\mathrm{M}$, et al. Store-operated Ca. Immunity. 2017;47(4):664-679.e6.

33. Monticelli S, Rao A. NFAT1 and NFAT2 are positive regulators of IL-4 gene transcription. Eur JImmunol. 2002;32(10):2971-2978.

34. Fowell DJ, et al. Impaired NFATc translocation and failure of Th2 development in Itk-deficient CD4+ T cells. Immunity. 1999;11(4):399-409.

35. Zenewicz LA, Antov A, Flavell RA. CD4 T-cell differentiation and inflammatory bowel disease. Trends Mol Med. 2009;15(5):199-207.

36. Mencarelli A, et al. Calcineurin B in CD4. Front Immunol. 2018;9:261.
37. Bootman MD. Calcium signaling. Cold Spring Harb Perspect Biol. 2012;4(7):a011171.

38. Raffaello A, Mammucari C, Gherardi G, Rizzuto R. Calcium at the center of cell signaling: interplay between endoplasmic reticulum, mitochondria, and lysosomes. Trends Biochem Sci. 2016;41(12):1035-1049.

39. Lam AK, Galione A. The endoplasmic reticulum and junctional membrane communication during calcium signaling. Biochim Biophys Acta. 2013;1833(11):2542-2559.

40. Mekahli D, Bultynck G, Parys JB, De Smedt H, Missiaen L. Endoplasmic-reticulum calcium depletion and disease. Cold Spring Harb Perspect Biol. 2011;3(6):a004317.

41. Grigoriu S, et al. The molecular mechanism of substrate engagement and immunosuppressant inhibition of calcineurin. PLOS Biol. 2013;11(2):e1001492.

42. Huang GN, et al. NFAT binding and regulation of $T$ cell activation by the cytoplasmic scaffolding Homer proteins. Science. 2008;319(5862):476-481.

43. Hogan PG. Calcium-NFAT transcriptional signalling in T cell activation and $\mathrm{T}$ cell exhaustion. Cell Calcium. 2017;63:66-69.

44. Martinez GJ, et al. The transcription factor NFAT promotes exhaustion of activated CD ${ }^{+} \mathrm{T}$ cells. Immunity. 2015;42(2):265-278.

45. Li HH, et al. Atrogin-1/muscle atrophy F-box inhibits calcineurin-dependent cardiac hypertrophy by participating in an SCF ubiquitin ligase complex. J Clin Invest. 2004;114(8):1058-1071.

46. Maejima Y, et al. Muscle-specific RING finger 1 negatively regulates pathological cardiac hypertrophy through downregulation of calcineurin A. Circ Heart Fail. 2014;7(3):479-490.

47. Shimizu H, et al. The calcineurin-FoxO-MuRF1 signaling pathway regulates myofibril integrity in cardiomyocytes. Elife. 2017;6:e27955.

48. Wang X, Huang Y, Li L, Wei Q. TRAF3 negatively regulates calcineurin-NFAT pathway by targeting calcineurin B subunit for degradation. IUBMB Life. 2012;64(9):748-756.

49. Wang DD, Wang HY, Zhou YH, Zhao CG, Dong C, Shuang SM. Study on the effects of cefotaxime on intracellular $\mathrm{Ca} 2+$ in human peripheral lymphocytes by fluoremetry. Chin Chem Lett. 2007;18(4):424-426.

50. Guichard V, et al. Calcium-mediated shaping of naive CD4 T-cell phenotype and function. Elife. 2017;6:e27215.

51. Mason MJ, Mahaut-Smith MP, Grinstein S. The role of intracellular $\mathrm{Ca} 2+$ in the regulation of the plasma membrane $\mathrm{Ca} 2+$ permeability of unstimulated rat lymphocytes. J Biol Chem. 1991;266(17):10872-10879.

52. Zinchenko VP, Antonov SA, Sergeev AI. Constitutively active calcium channels in plasma membrane of T-lymphocytes. Biochem (Mosc) Suppl Ser 
A Membr Cell Biol. 2009;3(3):286-290.

53. Navedo MF, Amberg GC, Votaw VS, Santana LF. Constitutively active L-type $\mathrm{Ca} 2+$ channels. Proc Natl Acad Sci U S A. 2005;102(31):11112-11117.

54. Suzuki Y, Inoue T, Ra C. L-type Ca2+ channels: a new player in the regulation of $\mathrm{Ca} 2+$ signaling, cell activation and cell survival in immune cells.
Mol Immunol. 2010;47(4):640-648.

55. Frangini $A$, et al. The aurora $B$ kinase and the polycomb protein ring1B combine to regulate active promoters in quiescent lymphocytes. Mol Cell. 2013;51(5):647-661.

56. Song J, Salek-Ardakani S, So T, Croft M. The kinases aurora $\mathrm{B}$ and $\mathrm{mTOR}$ regulate the G1-S cell cycle progression of T lymphocytes. Nat Immunol. 2007;8(1):64-73.

57. Uhlik M, et al. NF-kappaB-inducing kinase and IkappaB kinase participate in human $\mathrm{T}$-cell leukemia virus I Tax-mediated NF-kappaB activation. J Biol Chem. 1998;273(33):21132-21136. 